\title{
Assessing the Impacts of Augmented Observations on the Forecast of Typhoon Wutip's (2013) Formation Using the Ensemble Kalman Filter
}

\author{
Tran Tan Tien, Dao Nguyen-Quynh Hoa, and Cong Thanh \\ VNU University of Science, Vietnam National University, Hanoi, Vietnam
}

CHANH KIEU

Department of Earth and Atmospheric Sciences, Indiana University Bloomington, Bloomington, Indiana

(Manuscript received 2 January 2020, in final form 14 May 2020)

\begin{abstract}
In this study, the impacts of different augmented observations on forecasts of Typhoon Wutip's (2013) formation are examined. Using the local ensemble transformed Kalman filter (LETKF) implemented for the Weather Research and Forecasting (WRF) Model, it is found that the prediction of Wutip's formation location and timing is strongly governed by the strength of a monsoon trough that extends from the Bay of Bengal to the Philippine Sea. By properly capturing the strength of the monsoon trough after assimilating augmented observations available during Wutip's early stage, the WRF Model could provide better forecasts of Wutip's formation location and timing as compared to the forecasts initialized directly from global model analyses. Among different types of augmented observations, the satellite atmospheric motion wind vector (AMV) maintained by the Cooperative Institute for Meteorological Satellite Studies (CIMSS) appears to be the most critical in enhancing the large-scale representation of the monsoon trough. The benefit of augmented observations in Wutip's formation forecast is most apparent at about $36 \mathrm{~h}$ prior to the observed formation time. At the shorter 24-h lead time, there is, however, no clear benefit of augmented observations in predicting the timing and the location of Wutip's formation due to better global analyses. The results obtained in this study demonstrate the vital role of the CIMSS-AMV data in improving the large-scale environment required for TC formation that one should take into account for real-time TC forecasts.
\end{abstract}

\section{SIGNIFICANCE STATEMENT}

This study examines the sensitivity of tropical cyclone (TC) formation forecast to different types of local augmented observation for the case of Typhoon Wutip (2013). Results show critical impacts of satellite atmospheric motion vectors in enhancing large-scale environment where Typhoon Wutip's formation occurs, which is strongly controlled by a monsoon trough. The data impacts are optimal at $36 \mathrm{~h}$ prior to Wutip's formation and decrease as forecast cycles are closer to the observed formation time. In contrast, surface and local station data play a less significant role due to their scattered distributions that could not help strengthen the monsoon trough. These results highlight the importance of observations that can help improve the representation of large-scale environment for TC formation forecasting.

\section{Introduction}

Predicting tropical cyclone (TC) formation is one of the essential missions in current operational TC centers. While it is of practical importance, reliable forecast of TC formation has been difficult to accomplish so far due to our incomplete understanding of multiscale interaction of TCs with the ambient environment (e.g., Harr

Corresponding author: Dr. Tran Tan Tien, tientt49@gmail.com et al. 1996; Simpson et al. 1997; Halverson et al. 2007, Kieu and Zhang 2008, 2009; Wang et al. 2019). Despite great achievements in observing and studying tropical cyclogenesis in different ocean basins over the last few decades, a number of specific aspects of TC formation such as timing, location, or the duration of a cyclogenesis event are still challenging to predict at present, especially from the real-time forecast perspective (e.g., Chan and Kwok 1999; Cheung and Elsberry 2002; Halperin et al. 2013, 2016; Tallapragada et al. 2015; Chen et al. 2019). 
Generally, the key difficulties in predicting TC formation lie in the ad hoc nature of tropical cyclogenesis, which varies greatly from basin to basin. For example, observational and modeling studies of TC formation in the northwestern Pacific (WPAC) basin showed that tropical cyclogenesis is mostly related to the intertropical convergence zone (ITCZ) and monsoon activities in this basin (e.g., Yanai 1964; Gray 1968, 1982; Lander and Holland 1993; Harr et al. 1996; Wang et al. 2019). In contrast, TC formation in the North Atlantic basin often originates from active tropical waves (Avila and Pasch 1992; DeMaria 1996; Dunkerton et al. 2009). Because of this, the ad hoc nature of each individual basin has to be taken into account in forecast of TC formation.

In the WPAC basin, TC formation possesses some additional characteristics due to the strong influence of monsoon and complex terrain arrangement beyond traditional factors such as sea surface temperature (SST) or vertical wind shear (e.g., Gray 1968; Lander 1994; Carr and Elsberry 1995; Chan and Kwok 1999; Wang and Chan 2002; Wu et al. 2013; Dickinson and Molinari 2002; Frank and Roundy 2006; Cinco et al. 2016; Choi et al. 2017). Specifically, the low-level circulation in the WPAC basin is strongly governed by southwesterly wind from the Bay of Bengal and northeasterly wind from the Subtropical High during the TC main season (e.g., Li and Zeng 2002, 2003; Wang et al. 2004, 2008). With this unique monsoon influence, the WPAC basin is under constant competition between strong vertical wind shear and monsoonal circulations, resulting in a high variability in TC activity (Wang et al. 2010, Lin and Chan 2015, Wang and Chan 2002).

In addition to the strong influence of monsoonal systems, the part of the WPAC basin where TC activity can have potential impact on Vietnam's coastal region [i.e., defined in this study as an area over $5^{\circ}-25^{\circ} \mathrm{N}, 90^{\circ}-125^{\circ} \mathrm{E}$ and hereafter referred to as the domain of influence to Vietnam's coastal region (DOIV)] is also at the western edge of the WPAC basin with warm SST all year-round. This DOIV comprises almost the entire South China Sea (SCS) and a part of the Philippine Sea. As a result of climatologically warmer SST during the peak months from May to October in this region (e.g., Tan et al. 2016), TC climatology in the DOIV is modulated by many factors beyond the traditional proxies. On average, there are about 5-7 TCs in this region that can pose either direct or indirect threat to Vietnam's coastline every year. Thus, having a reliable early warning of TC formation and subsequent development is of great importance to Vietnam's economy and risk management.

Due to the unique geography of the DOIV as well as a scattered distribution of observing networks in this area, predicting TC formation in the DOIV, particularly those that are in close proximity to Vietnam's coastal region, is very challenging even for 1-3-day lead times. Among several approaches for real-time monitoring and forecasting of TC formation, direct numerical products from global and regional weather prediction models appear to be the most reliable at present, despite their inherent limitations and uncertainties (e.g., Zhao et al. 2009; Chen et al. 2019). The skillful performance of TC formation forecasts by numerical models has been well documented in many previous studies (Chan and Kwok 1999; Oouchi et al. 2006; Cheung and Elsberry 2002; Halperin et al. 2013, 2016; Tallapragada et al. 2015). This noticeable skill of numerical models is attributed to various improvements in model physics, resolution, computational scheme, and data assimilation schemes. The role of data assimilation is especially noteworthy here, given the constant implementation of new observational types and networks that help better represent the initial state of the atmosphere in current operational modeling systems (Zhou et al. 2019; Chen et al. 2019).

Among several different assimilation schemes, the ensemble Kalman filter (EnKF) has been extensively applied to many practical problems in recent years due to its straightforward implementation for TC forecast applications (Snyder and Zhang 2003; Zhang et al. 2011; Zhang and Weng 2015; Holt et al. 2015; Kieu et al. 2014; Aberson et al. 2015; Tong et al. 2018). The use of EnKF for TC forecasting applications is increasingly popular, given the current availability of real-time flight reconnaissance data that allows direct assimilation of airborne observations without the need of a bogus vortex (see, e.g., Zhang and Weng 2015; Aberson et al. 2015; Tong et al. 2018; Zhang et al. 2011). An important advantage of the EnKF method as compared to variational assimilating schemes is that the background covariance matrix is allowed to vary in time, thus adapting better with fast-evolving dynamical systems such as TCs or mesoscale convective systems (Snyder and Zhang 2003; Pu et al. 2016; Kieu et al. 2014; Du et al. 2017).

Given the difficulty in predicting tropical cyclogenesis in the DOIV that often occurs in a short period of time we wish to examine in this study the impacts of several types of augmented observational data on timing and location of Typhoon Wutip's (2013) formation. This typhoon first emerged from a tropical disturbance in the SCS on 23 September 2013, and quickly evolved into a typhoon after just 1 day. Such a rapid development and a short lifetime of Wutip is fairly common for TC formation in the DOIV and always poses a challenge in realtime forecasts (e.g., Wei et al. 2016; Zhong et al. 2018; X. Zhang et al. 2018). Unlike traditional TC track and intensity forecasts for which TC centers are well defined at the initial time, TC genesis forecasting possesses several 


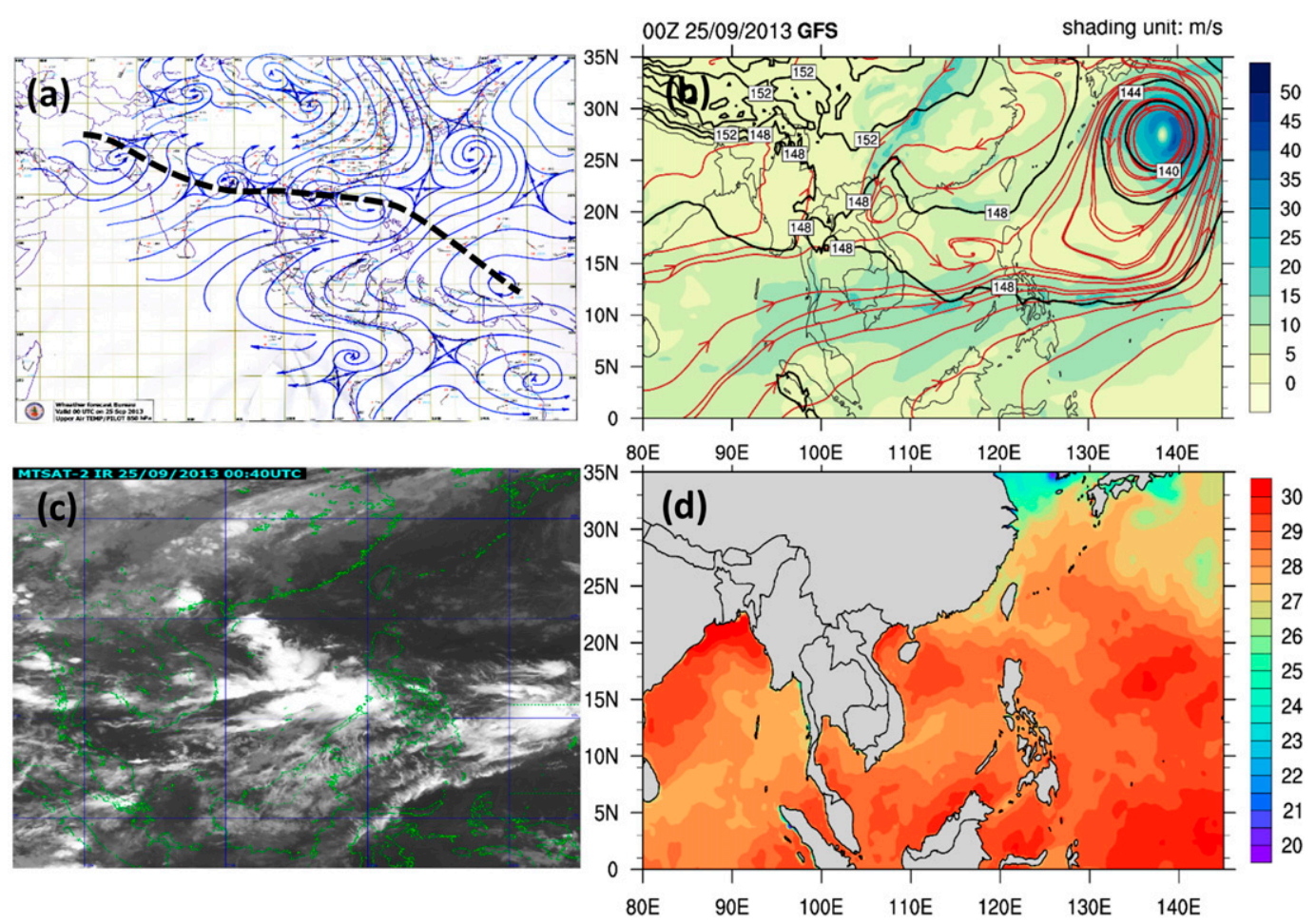

FIG. 1. (a) Synoptic analyses of observed surface streamline (contours) valid at 0000 UTC 25 Sep, (b) the largescale flow (green lines) and geopotential perturbation (red lines) at $850 \mathrm{hPa}$ obtained from the GFS analyses valid at 0000 UTC $25 \mathrm{Sep}$, (c) MTSAR-2 IR satellite image of cloud disturbances valid at 1140 UTC 25 Sep when Wutip first formed near the Philippine Archipelago, and (d) the SST distribution (shaded). The thick dashed line in (a) denotes the monsoon trough associated with Typhoon Wutip's formation.

distinct characteristics that depend largely on ambient environment. Specifically, one has to focus on unknown locations and time where an early tropical depression may form instead of TC-following motion and intensity. Thus, the data assimilation approach based on target observations as for typical track and intensity forecasts cannot be applied for real-time purposes. Our main objective of this study is to explore how the forecast of Wutip's formation responds to different types of available augmented observations during its early stage, using a variant of the EnKF, the so-called local ensemble transformed Kalman filter (LETKF) scheme.

The remainder of the paper is organized as follows. In the next section, an overview of Typhoon Wutip (2013) will be provided. Details of model experiments, data assimilation techniques, and data input are presented in section 3. Section 4 discusses the main results related to the timing and location forecast of Wutip's formation, and conclusions are given in the final section.

\section{Overview of Wutip}

Typhoon Wutip (2013) originated from a low pressure system in the DOIV around 1200 UTC 25 September
2013. It was embedded within a preexisting monsoon trough that extended from the Bay of Bengal to the Philippines Sea (Fig. 1a). A low pressure system located around $14^{\circ} \mathrm{N}, 118^{\circ} \mathrm{E}$ gradually strengthened into a tropical depression around $720 \mathrm{~km}$ east of the Paracel Islands at 1800 UTC 25 September with the strongest wind near the center around $\sim 11-14 \mathrm{~m} \mathrm{~s}^{-1}$ (category 6-7) according to the Beaufort scale. The depression moved gradually in the northwest direction with little development until 0000 UTC 27 September, when it moved over a warm SST pool. In just about $9 \mathrm{~h}$, the depression quickly grew and strengthened to a tropical storm (category 8) at 0700 UTC 27 September (Fig. 2). By 1900 UTC 28 September, Wutip fully developed into a typhoon at roughly $17.5^{\circ} \mathrm{N}, 114.5^{\circ} \mathrm{E}$ and started to move westward at speed of about 2-3 $\mathrm{m} \mathrm{s}^{-1}$. Typhoon Wutip made landfall in central Vietnam in the afternoon of 30 September with the maximum sustained winds of category 11, causing significant damage to Vietnam's coastal region.

The rapid development of Wutip from a tropical depression to a typhoon in just about $23 \mathrm{~h}$ posed serious difficulty to local forecasters. In fact, the early signal of Wutip in the global model input was very weak (Fig. 1b), 

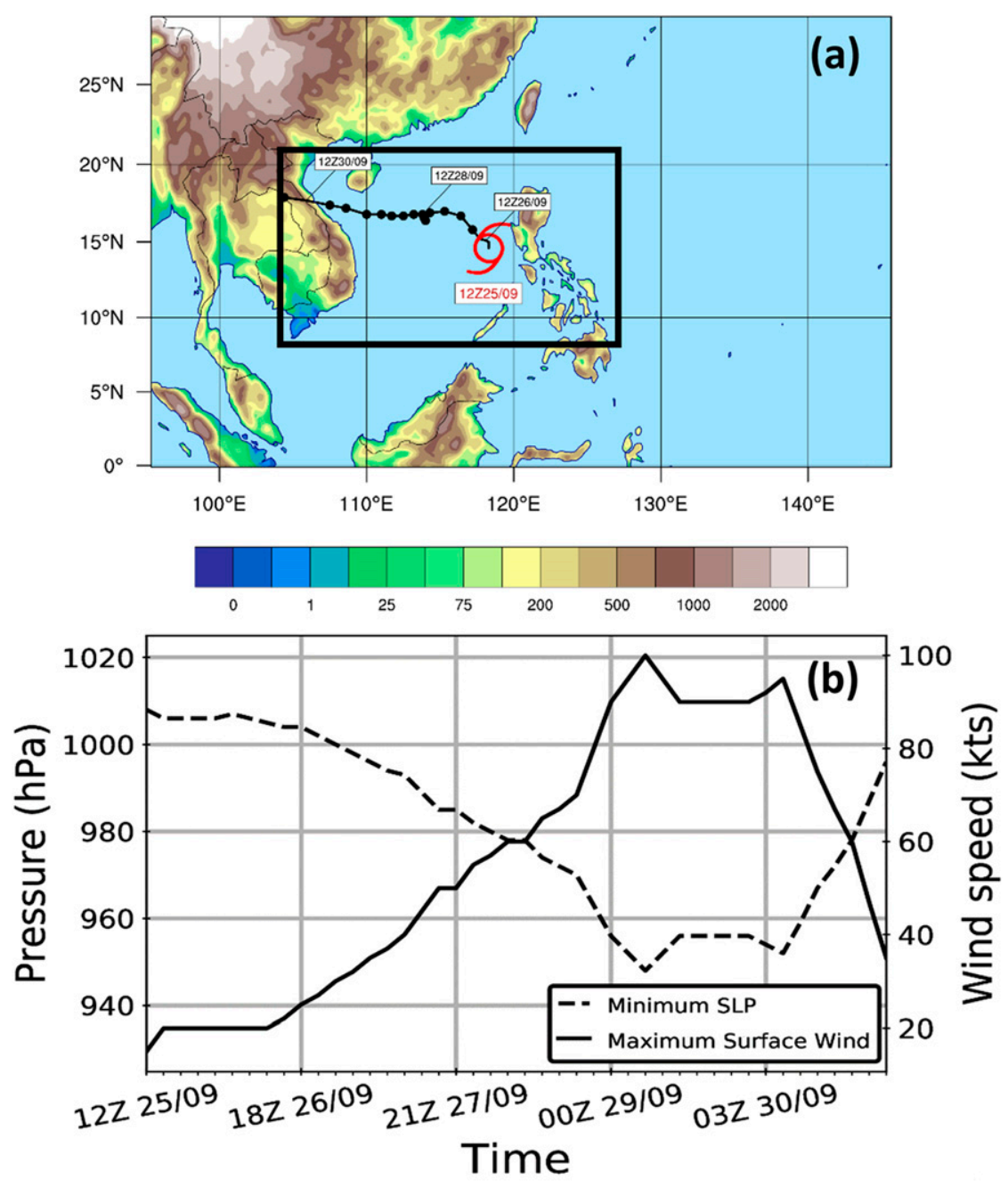

FIG. 2. (a) The domain configuration for the WRF-LETKF system that is designed for forecasting TC formation, which consists of an outer domain at a resolution of $27 \mathrm{~km}$ and a domain of influence (DOIV) at a resolution of $9 \mathrm{~km}$ that is nested within the outer domain (a black box); the terrain height ( $\mathrm{m}$ ) is shaded. (b) Typhoon Wutip's time series of the maximum 10-m wind (solid), and minimum central pressure (dashed) from 1200 UTC 25 Sep. Superimposed in (a) is the observed track of Typhoon Wutip during its recorded lifetime.

and it became noticeable about just 2 days before its landfall. In particular, Wutip's formation was too close to Vietnam's coastline before it could be fully captured in numerical models, thus giving forecasters very little time to issue an effective warning and risk announcement. The above development of Wutip is one of several typical tropical cyclogenesis patterns in the WPAC, where a monsoon trough strongly interacts with a continental high pressure system over Mainland China. In fact, there were multiple TCs during this active phase of the monsoon activity from 12 to 31 September including Typhoon Usagi, Tropical Storm 18W, Tropical Storm Pabuk, Typhoon Wutip, and Tropical Storm Sepat. In this regard, predicting such an early TC formation associated with the monsoon trough activities is critical, especially the timing and the location of the main development area. This question is of importance for not only improving the prediction of early TC formation, but also for planning observational networks that are most critical and effective for TC prediction in the future.

\section{Experiment design}

\section{a. Model description}

To examine Wutip's formation, the nonhydrostatic version of the Weather Research and Forecast (WRFARW) Model (V3.6; Skamarock et al. 2005) is used in 
TABLE 1. List of all combinations of physical parameterization schemes used in the WRF-LETKF system with a multiple-physics ensemble design.

\begin{tabular}{|c|c|c|c|c|}
\hline Member & $\begin{array}{c}\text { Cumulus } \\
\text { parameterization }\end{array}$ & $\begin{array}{c}\text { Microphysics } \\
\text { parameterization }\end{array}$ & $\begin{array}{l}\text { Boundary layer } \\
\text { parameterization }\end{array}$ & $\begin{array}{c}\text { Shortwave radiative } \\
\text { parameterization }\end{array}$ \\
\hline 1 & Kain-Fritsch & Kessler & YSU & Goddard \\
\hline 2 & Betts-Miller-Janjić & Kessler & YSU & Dudhia \\
\hline 3 & Betts-Miller-Janjć & Lin et al. & YSU & Goddard \\
\hline 4 & Grell-Freitas & Lin et al. & YSU & Dudhia \\
\hline 5 & Grell-Freitas & WSM 3 & YSU & Goddard \\
\hline 6 & Kain-Fritsch & WSM 3 & YSU & Dudhia \\
\hline 7 & Kain-Fritsch & Kessler & MYJ & Goddard \\
\hline 8 & Betts-Miller-Janjić & Kessler & MYJ & Dudhia \\
\hline 9 & Betts-Miller-Janjić & Lin et al. & MYJ & Goddard \\
\hline 10 & Grell-Freitas & Lin et al. & MYJ & Dudhia \\
\hline 11 & Grell-Freitas & WSM 3 & MYJ & Goddard \\
\hline 12 & Kain-Fritsch & Kessler & MRF & Dudhia \\
\hline 13 & Kain-Fritsch & Kessler & MRF & Goddard \\
\hline 14 & Betts-Miller-Janjić & Lin et al. & MRF & Dudhia \\
\hline 15 & Betts-Miller-Janjić & WSM 3 & MRF & Goddard \\
\hline 16 & Grell-Freitas & Kessler & YSU & Dudhia \\
\hline 17 & Grell-Freitas & Kessler & YSU & Goddard \\
\hline 18 & Grell-Freitas & Lin et al. & YSU & Dudhia \\
\hline 19 & Kain-Fritsch & WSM 3 & YSU & Goddard \\
\hline 20 & Betts-Miller-Janjić & WSM 3 & YSU & Dudhia \\
\hline 21 & Betts-Miller-Janjić & WSM 3 & YSU & Goddard \\
\hline
\end{tabular}

this study. The WRF Model is designed with an outermost domain at the horizontal grid spacing of $27 \mathrm{~km}$ and a nested domain at resolution of $9 \mathrm{~km}$ with 31 vertical levels. This outermost domain covers an area of $95^{\circ}-145^{\circ} \mathrm{E}, 0^{\circ}-30^{\circ} \mathrm{N}$ in the west-east and north-south directions, which is sufficiently large to cover the entire DOIV and a part of the northwest Pacific basin for our TC genesis study (Fig. 2).

It should be noted that unlike TC intensity forecasts for which the capability to resolve the inner-core region is important, TC formation is often largely governed by the large-scale environment (e.g., Chan and Kwok 1999; Chan and Xu 2009; Cheung and Elsberry 2002; Strachan et al. 2013; Halperin et al. 2013, 2016; Zhou et al. 2019; Chen et al. 2019). The fact that current global models at a coarser resolution such as the operational National Centers for Environmental Prediction (NCEP) Global Forecasting System (GFS) or the European Centre for Medium-Range Forecasting (ECMWF) model could have a high success rate of cyclogenesis prediction indicates that the ambient environment is crucial for TC genesis forecast. Thus, the 27-/9-km resolutions are adopted here as a compromise between computational resources required for our operational ensemble data assimilation experiments and the characteristics of TC formation that we are examining.

For the model physics, a suite of physical parameterization schemes available in the WRF Model is used in our ensemble experiments, following the multiple-physical ensemble approach in Kieu et al.
(2014; Du et al. 2017). Unlike ensemble systems with a single set of model physics schemes (e.g., Tong et al. 2018; Zhang et al. 2015), previous studies demonstrated that the use of multiple parameterization schemes for ensemble forecasts could help significantly improve the ensemble spread and related forecast skill. In this study, the set of physical schemes used for our ensemble experiments are 1) two cumulus parameterization schemes including the Betts-Miller-Janjić (BMJ) cumulus parameterization and the Kain-Fritsch with shallow convection schemes, 2) three planetary boundary layer (PBL) parameterization schemes including the Yonsei University, the Mellor-Yamada-Janjić, and the simple Medium-Range Forecast (MRF) schemes, 3) three microphysical schemes including the WSM 3 microphysics, the Kessler, and the Lin et al. schemes; and 4) two longwave radiative schemes including the Dudhia and the Goddard schemes for both longwave and shortwave radiations. While a total of 36 different possible combinations of these physical schemes can be formed, many combinations are either not allowed due to some internal constraint among different parameterization schemes or cause numerical instability during integration. Of all possible combinations, only 21 combinations can be therefore used for real-time purposes based on their numerical stability (see Table 1 for the list of the combinations and related discussion in Kieu et al. 2014). If the number of ensemble members is larger than the number of the combinations, 
the assignment will be repeated for the next ensemble members.

\section{b. Data assimilation system}

In this study, the LETKF algorithm proposed by Ott et al. (2004) and Hunt et al. (2007) is adopted and implemented for the WRF Model to study the formation of Typhoon Wutip. The main advantage of the LETKF algorithm is to use the background ensemble matrix as an operator that transforms state vectors from a model space spanned by the model grid points within a local patch to an ensemble space spanned by ensemble members. All matrix calculations and generation of the ensemble analyses are then carried out in this lowdimension ensemble space at each grid point. Such a local approach in the ensemble space allows an efficient parallelization, which is particularly appealing for rapid processing a large-volume of data for practical problems (e.g., Kang et al. 2011; Szunyogh et al. 2008; Yang et al. 2009; Miyoshi and Kunii 2012; Kieu et al. 2012, 2014; Holt et al. 2015).

Because of its promising capability, LETKF has been implemented in the WRF Model (V3.6, hereafter referred to as the WRF-LETKF system) for typhoon forecasting at the Vietnam Hydro-Meteorological Office. As a step toward practical forecasting applications, the WRF-LETKF system is designed in such a way that all observations are subject to a quality control by the WRF data assimilation (WRFDA) component before used by the LETKF algorithm. In addition, a utility provided by the WRFDA is used to generate the lateral boundary conditions for each ensemble member once the analysis update is obtained. By doing this, each ensemble member possesses its own boundary dynamically consistent with its own analysis. More details about the implementation of the WRF-LETKF design can be found in Kieu et al. $(2012,2014)$.

Due to our limited computational and storage resources, all ensemble experiments in this study have a fixed number of 21 ensemble members, which is the same number of possible combinations of different physical schemes in the WRF Model. As discussed in Kieu et al. (2012), this design of ensemble members optimizes the ensemble spread without the expensive cost related to adding more ensemble members. Although 21 members appear to be relatively small, our main focus here is on the relative performance of ensembles with and without augmented observations. In this regard, the relative differences between these ensembles can still provide the main effects of augmented observations.

To initialize the ensemble system, a cold-start background ensemble is generated by first using the 3DVAR scheme to create an analysis from a given GFS initial condition. Random perturbations with standard deviations of $1 \mathrm{~m} \mathrm{~s}^{-1}$ for the wind field, $1 \mathrm{~K}$ for temperature, and $1 \times 10^{-3} \mathrm{~kg} \mathrm{~kg}^{-1}$ for specific humidity at all model grid points are then added to the 3DVAR-generated analyses for the cold-start ensemble. This cold-start ensemble is then integrated for $12 \mathrm{~h}$ such that the outputs from these 12-h integrations can be subsequently used as a warm-start background for the LETKF ensemble assimilation next cycle. Note that these random perturbations are added only for the first cold-start cycle to initialize a background ensemble. All subsequent cycles use the WRF-LETKF 12-h forecasts as a background ensemble and so no random noises are required. The newly generated analysis perturbation ensemble at each cycle is then added to the GFS analysis to produce the next ensemble initial conditions when run in the cycling mode as described in Du et al. (2017).

Given our focus on the prediction of Wutip's early formation, all ensemble experiments are integrated for three days starting from 1200 UTC 23 September, which is $\sim 48 \mathrm{~h}$ before a tropical depression precursor of Wutip was first reported in the TC vital record at 1200 UTC 25 September. For the case of Typhoon Wutip, the coldstart cycle is therefore initialized at 0000 UTC 23 September to generate a background ensemble for the first data assimilation cycle at 1200 UTC 23 September. The entire WRF-LETKF data assimilation system is subsequently cycled every $6 \mathrm{~h}$ from 1200 UTC 23 September to 1200 UTC 26 September.

\section{c. Observation and input data}

Given our focus on examining the real-time TC formation, the initial and boundary conditions in all ensemble experiments in this study are taken from the global NCEP GFS operational forecasts at a horizontal resolution of $0.5^{\circ} \times 0.5^{\circ}$ that are updated in real time every $6 \mathrm{~h}$. In addition to this 6-h boundary condition update, all integrations are configured with SST updated every $6 \mathrm{~h}$ to increase the response of TC development to the actual SST condition. This SST field is extracted directly from the GFS final analyses that are also available at the NCEP real-time product portal. ${ }^{1}$

For the augmented observational data used in the LETKF assimilation, two main sources of observations are employed in this study. The first is the satellitederived atmospheric motion vector (AMV) data maintained by the Cooperative Institute for Meteorological Satellite Studies (CIMSS), University of Wisconsin

\footnotetext{
${ }^{1}$ Real-time GFS forecast can be accessed at https://ftp.ncep.noaa.gov/ data/nccf/com/gfs/prod/.
} 
(a)

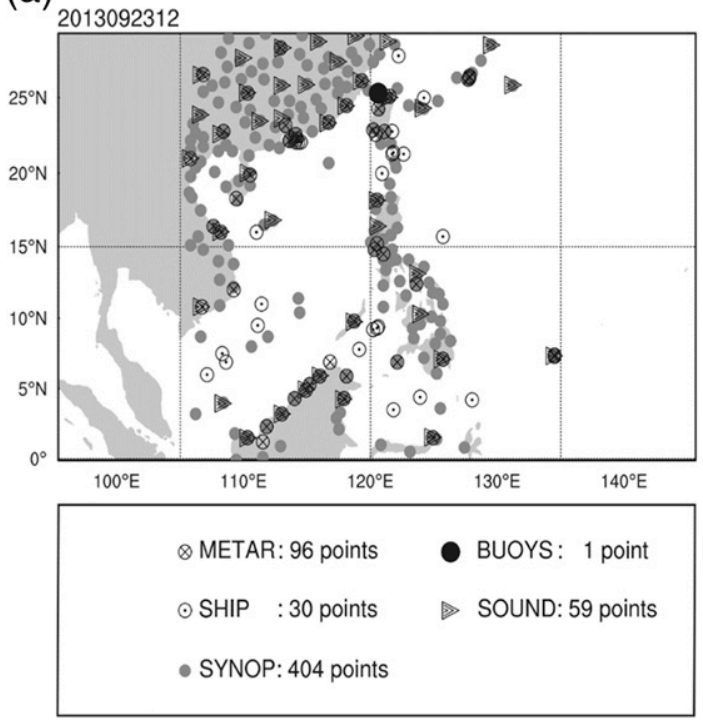

(b)

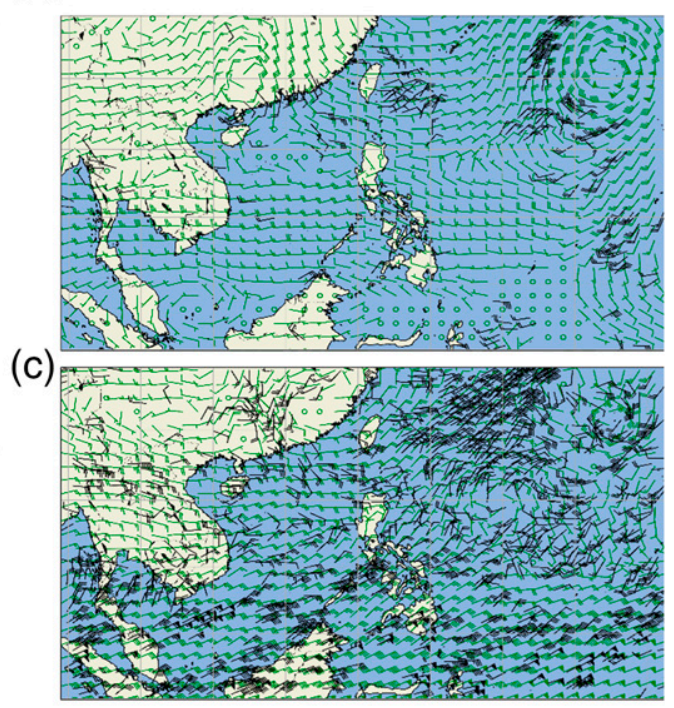

FIG. 3. (a) Locations of all available augmented observations based on the station, buoy, sounding, METAR, and ship that are used in the WRF-LETKF system during the forecast of Wutip's formation; (b) the AMV data distribution (black barbs) in the 800-300-hPa layer; and (c) as in (b), but for the 300-80-hPa layer. Superimposed is the GFS initial condition wind field (green barbs).

(Velden et al. 1992, 1997; Holmlund et al. 2001; Le Marshall et al. 2004; Li et al. 2020). By default, the CIMSS AMV satellite database is divided into several different ocean basins in the ASCII format that can be readily processed and reformatted for further dataquality check and assimilation. For our TC formation study, the CIMSS-AMV satellite data in the WPAC basin is especially beneficial, because this data covers a large area where TC genesis may take place. While the CIMSS AMV data should have its quality control threshold depending on the wind magnitude and levels as discussed in $\mathrm{Wu}$ et al. (2014, 2015), S. Zhang et al. (2018), or Li et al. (2020), we adopt a simple approach for our WRF-LETKF system in which the observation error for the AMV wind is set equal to $3.5 \mathrm{~m} \mathrm{~s}^{-1}$ and the corresponding quality indicator is $>0.7$. This choice of a constant wind error vector and a fixed quality indicator is merely to retain more AMV data and its impacts in the DOIV subdomain (see Figs. 3b,c). Details of the quality control and expected errors for the AMV data can be found in Velden et al. (1992, 1997), Holmlund et al. (2001), or Wu et al. $(2014,2015)$.

Along with the AMV satellite data, the second source of local augmented observations in the DOIV is also used. These local data include 96 aviation routine weather (METAR) reports from routine scheduled observations, 31 ship/buoy station reports, 59 enhanced sounding stations, and 404 surface synoptic observations (SYNOP) reports of weather observations during the 0000 UTC 24 September-0000 UTC 27 September period. These local observations are not regularly distributed (see Fig. 3) and contain a mix of various data types such as time of observation, wind, visibility, present weather phenomena, sky conditions, temperature, dewpoint, and altimeter setting. While some of the SYNOP data were already distributed and shared to all global and regional centers, we note that most local stations along Vietnam's coastline and the METAR data were only available after 1200 UTC 23 September, which is roughly $48 \mathrm{~h}$ before the formation of Wutip. Because these augmented observations are standard, the observation errors for these surface and radiosonde data are directly taken from WRFDA, which provides an observation preprocessor (OBSPROC) utility and a default observation error table for all standard data types.

For the sake of discussion, all forecast cycles in this study will be hereafter referred to as 48-, 36-, or 24 -h cycles, meaning that the forecast cycles are initialized 48 , 36 , or $24 \mathrm{~h}$ prior to the observed formation of Wutip reported in the best track data.

\section{d. Vortex tracking method}

Given the model output from WRF-LETKF ensemble forecasts, a vortex-tracking algorithm is necessary to detect the formation of a newly developed TC center in all ensemble members. A good vortex detection scheme is critical for the TC formation study such that the timing 
and the location of TC formation centers can be properly captured and verified. Due to the undefined structure of tropical disturbances during the early genesis stage, determining their vortex center for TC genesis forecast is generally difficult. Specifically, one cannot apply common criteria such as a midlevel warm core anomaly, storm inner and outer sizes, or maximum vorticity centers for the genesis stage as for mature TCs. In fact, the early formation of a tropical depression is often marked by the existence of an upper-level cold core and a weak surface low pressure rather than a midlevel warm core (see, e.g., Bister and Emanuel 1997; Wen et al. 2018). Thus, very few conditions can be practically applied to detect a formation center during the genesis stage.

To detect TC formation centers for real-time forecast purposes in this study, we adopt a simple scheme that is based on the most basic conditions related to the maximum surface wind and the minimum center pressure as follows:

- First, the minimum sea level pressure $P_{\min }$ within the DOIV is searched at every model grid point of each ensemble member output for a given forecast lead time. Any location with $P_{\min }<1004 \mathrm{hPa}$ will be marked as a potential candidate for TC formation location at that forecast lead time for that ensemble member.

- Second, once a location for possible TC formation is found, the maximum $10-\mathrm{m}$ wind speed $V_{\max }$ in an area of $4^{\circ} \times 4^{\circ}$ around the minimum pressure center is checked and recorded. A TC formation center will be marked if the condition $V_{\max } \geq 10 \mathrm{~m} \mathrm{~s}^{-1}$ is satisfied. We note that this value is considerably smaller than the definition of a tropical depression wind speed $\left(\sim 17 \mathrm{~m} \mathrm{~s}^{-1}\right)$. However, this value is adopted here due to the relatively coarse $27-/ 9-\mathrm{km}$ resolution configuration of our WRF-LETKF system. Our manual verification of each circulation center detected from model output based on this threshold confirms that these criteria can properly capture the center of TC circulation during the genesis stage. Therefore, this threshold for $V_{\max }$ is used for all genesis analyses in this study.

While these criteria for tracking TC formation centers are somewhat subjective, this simple approach is sufficiently for our purpose of evaluating the data impacts on $\mathrm{TC}$ formation forecasts among different ensembles. So long as these tracking criteria are the same in all analyses, the comparison of $\mathrm{TC}$ formation forecasts should provide good information of how augmented observation impacts on the ensemble forecasts that we wish to present in this study.

\section{Results}

\section{a. Initial condition analyses}

To gain a broad assessment of the improvement in the large-scale environment after assimilating augmented observations prior to the formation of Typhoon Wutip, Figs. $4 \mathrm{a}$ and $4 \mathrm{~b}$ shows horizontal cross sections of the initial ensemble means of geopotential perturbation and streamline at $700 \mathrm{hPa}$ from the WRF-LETKF system (hereafter referred to as the control, or CTL, ensemble). Also displayed are horizontal cross sections obtained directly from the GFS initial data [hereafter to as no data assimilation (NDA) ensemble] valid at 1200 UTC 23 September. This cycle is approximately $48 \mathrm{~h}$ before the first report of Wutip's tropical depression at 1200 UTC 25 September. Note that geopotential perturbation and horizontal winds are two prognostic variables in the WRF Model that are directly assimilated by the WRF-LETKF system. Thus, their differences between the CTL and the GFS initial conditions are analysis increments, which demonstrate directly the impacts of data assimilation. Although the NDA ensemble use the same GFS initial and boundary conditions for all members and does not assimilate any augmented observations, it should be mentioned that the NDA ensemble are still configured with multiple physical schemes similar to the CTL ensemble. This multiple-physics design for the NDA ensemble is needed such that the comparison between the CTL and NDA ensembles can better indicate the effect of augmented observations.

One notices first in Figs. 4a and 4b an apparent signal of a monsoon trough with noticeable northeasterly wind on the northern part and southwesterly wind on the southern part of the DOIV in both the CTL and NDA initial conditions. Except for some local influence of the high topography in the Central Highlands of Vietnam and mainland China, both initial conditions capture also an elongated band of low pressure along the monsoon trough where horizontal wind converges (cf. Fig. 1a). Despite these overall similar large-scale atmospheric patterns, more detailed comparison of these initial conditions shows that there are several important differences between the two initial conditions as displayed by analysis increments of geopotential perturbation and wind field (Figs. 4c,d). Indeed, assimilating augmented observations produces a significant enhancement of the low pressure band along the monsoon trough over the DOIV, and another low pressure area to the north of the Philippine Sea that is associated with a nearby Typhoon Usagi (2013). Corresponding to the enhanced monsoon trough are the increments of converging winds 

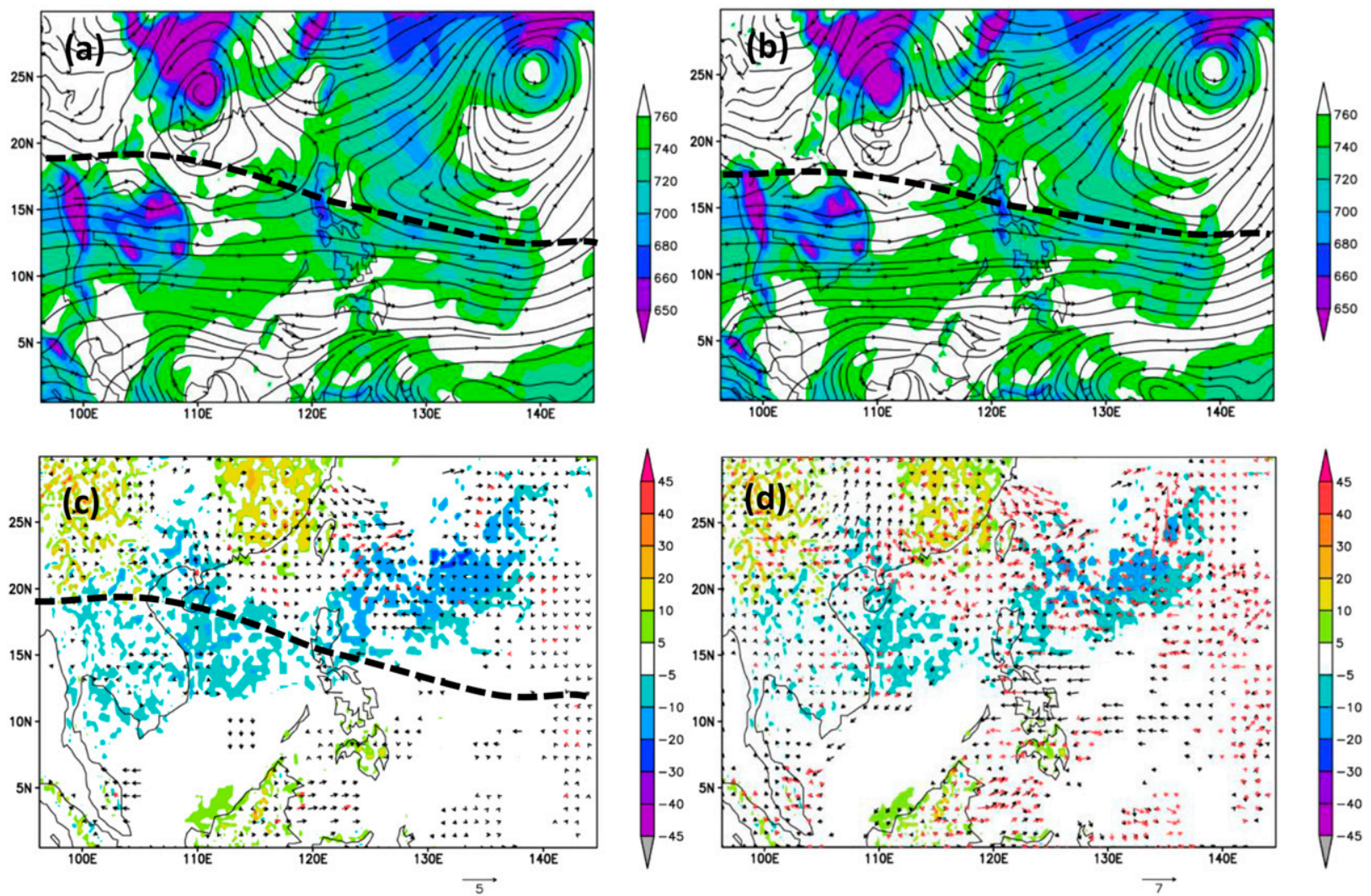

FIG. 4. Horizontal cross section at the initial time for the 1200 UTC 23 Sep cycle of geopotential height perturbation $\left(\right.$ shaded; $\mathrm{m}^{2} \mathrm{~s}^{-2}$ ) and the corresponding streamline at $700 \mathrm{hPa}$ obtained from (a) the WRF-LETKF system; (b) the GFS initial input; and (c) analysis increment (CTL-GFS) of the geopotential perturbation (shaded) and the analysis increment of the wind vectors (black) as obtained from the WRF-LETKF system at $700 \mathrm{hPa}$, superimposed by the corresponding observational wind increment (red vectors); and (d) as in (c), but for the 200-hPa level. The thick dashed line denotes the location of the monsoon trough in the lower-tropospheric layer.

toward the trough and cyclonic winds related to Typhoon Usagi, which is consistent with the observational wind increments (Figs. 4b-d). Despite higher density of the AMV data at the upper level, the enhancement of monsoon trough in terms of geopotential increments is less apparent at the upper troposphere (Fig. 4d) as compared to the lower levels (Fig. 4c) where the monsoonal influence is often stronger as expected.

Similar analyses for the next two cycles at 0000 and 1200 UTC 24 September, which are $\sim 36$ and $24 \mathrm{~h}$ before the formation of Wutip's depression, are shown in Fig. 5. One notices again the emergence of a low pressure anomaly extending from the far west of the Vietnam Central Highlands to the Philippine Sea after assimilating augmented observations, which are most apparent at the lower level shown in Fig. 5. This persistent enhancement of this low pressure band along the monsoon trough for the 48- and 36-h cycles as a result of assimilating augmented observations confirms the weaker strength of the monsoon trough in all GFS initial condition until 1200 UTC 24 September. By 1200 UTC
24 September, note that the analysis increment of geopotential height is significantly reduced, indicating that the GFS initial conditions could now better capture the strength of the monsoon trough about $24 \mathrm{~h}$ prior to Wutip's formation. On the one hand, a lower pressure band consistently enhanced along the monsoon trough in all CTL initial conditions strongly suggests the underestimation of GFS initial analyses in capturing the correct monsoon strength during the early stage of Wutip's formation. On the other hand, this persistence of such a low pressure increment highlights that the LETKF system is effective in enhancing the representation of large-scale flows as expected.

From the physical perspective, the development of negative geopotential increments over a large ocean area is a result of dynamical consistency between the mass and the wind fields in the WRF-LETKF system. We recall that most of augmented observations in the CTL ensemble are related to the AMV data over the open ocean, due to the broad coverage of moving clouds. While the majority of the AMV data are at higher levels 

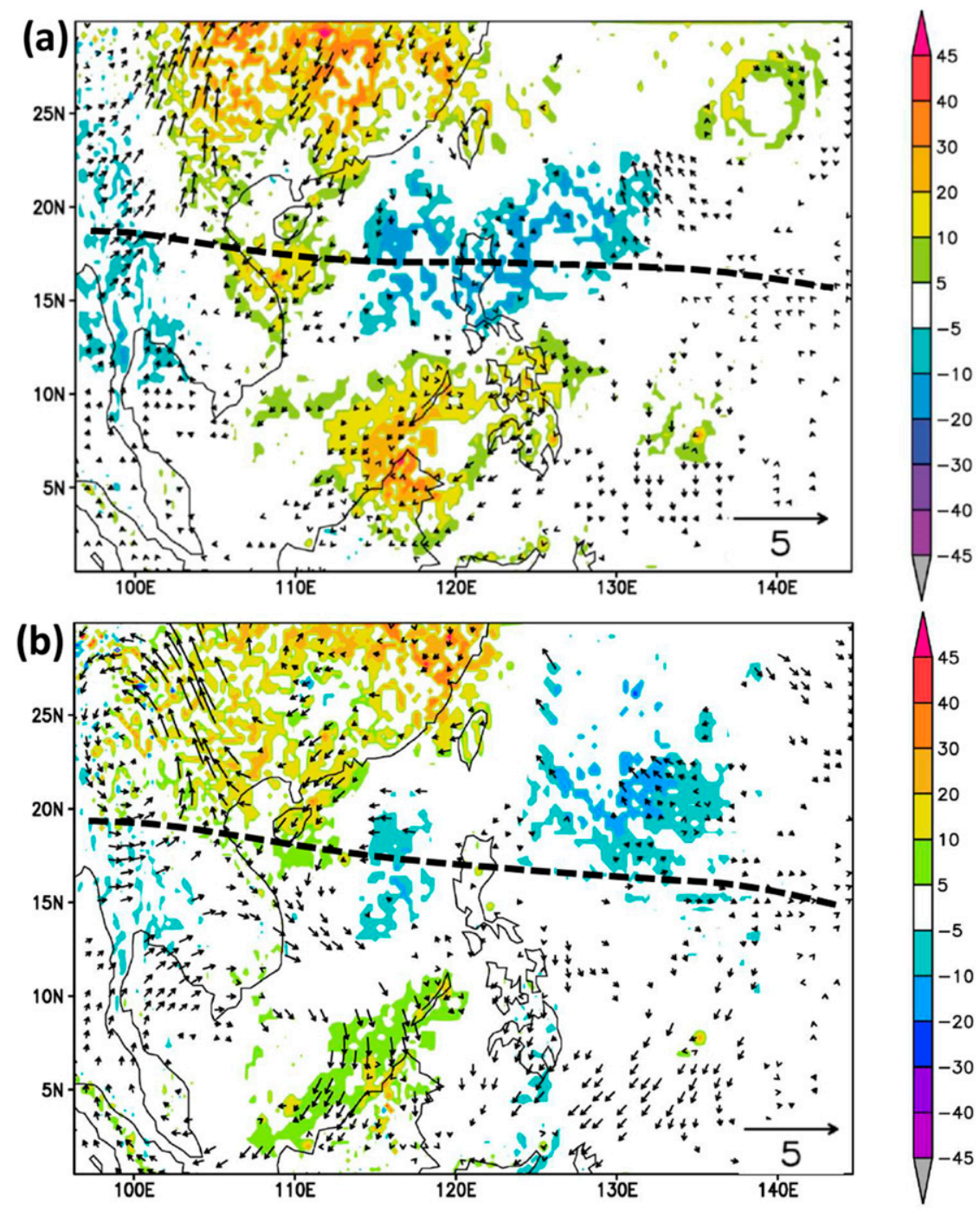

FIG. 5. Analysis increment (CTL-GFS) of the geopotential perturbation (shaded) and the analysis increment of the wind vectors (black) as obtained from the WRF-LETKF system at $700 \mathrm{hPa}$ for the cycle (a) 0000 UTC 14 Oct and (b) 1200 UTC 14 Oct 2013, which is about 36- and 24-h prior to the formation of Wutip's depression.

(above $450 \mathrm{hPa}$, see Figs. $3 \mathrm{c}$ and $3 \mathrm{~d}$ ) due to the need of tracking cloud motion, the cross correlation between the wind and the mass fields developed within the WRFLETKF system could spread the wind information to geopotential field as seen in Figs. 4 and 5. That is, the balance constraint in the WRF-LETKF system imposes a condition on the mass and the wind fields via a cross correlation among ensemble members, thus inducing a low pressure anomaly that corresponds to enhanced large-scale cyclonic flows. The fact both the cyclonically enhanced wind field and the negative geopotential anomaly appear after assimilating augmented observations indicates that the CTL ensemble could develop some internal constraint along the monsoon trough as expected.
Given the distribution of augmented observation locations in Fig. 3 and the corresponding analysis increments in Fig. 4, it is anticipated that much of the analysis increments along Vietnam's coastal region should be attributed to the dense concentration of local station data. To isolate this role of the AMV data versus other station/buoy observations, Fig. 6 displays analysis increments of geopotential perturbation and wind field for two sensitivity experiments in which no CIMSS-AMV data are assimilated in Fig. 6a (NAMV), and no station data are used in Fig. $6 \mathrm{~b}$ for the 1200 UTC 23 September cycle. One notices apparently from these figures the effects of the AMV data, which help enhance both the wind and the mass fields over a much larger area than 

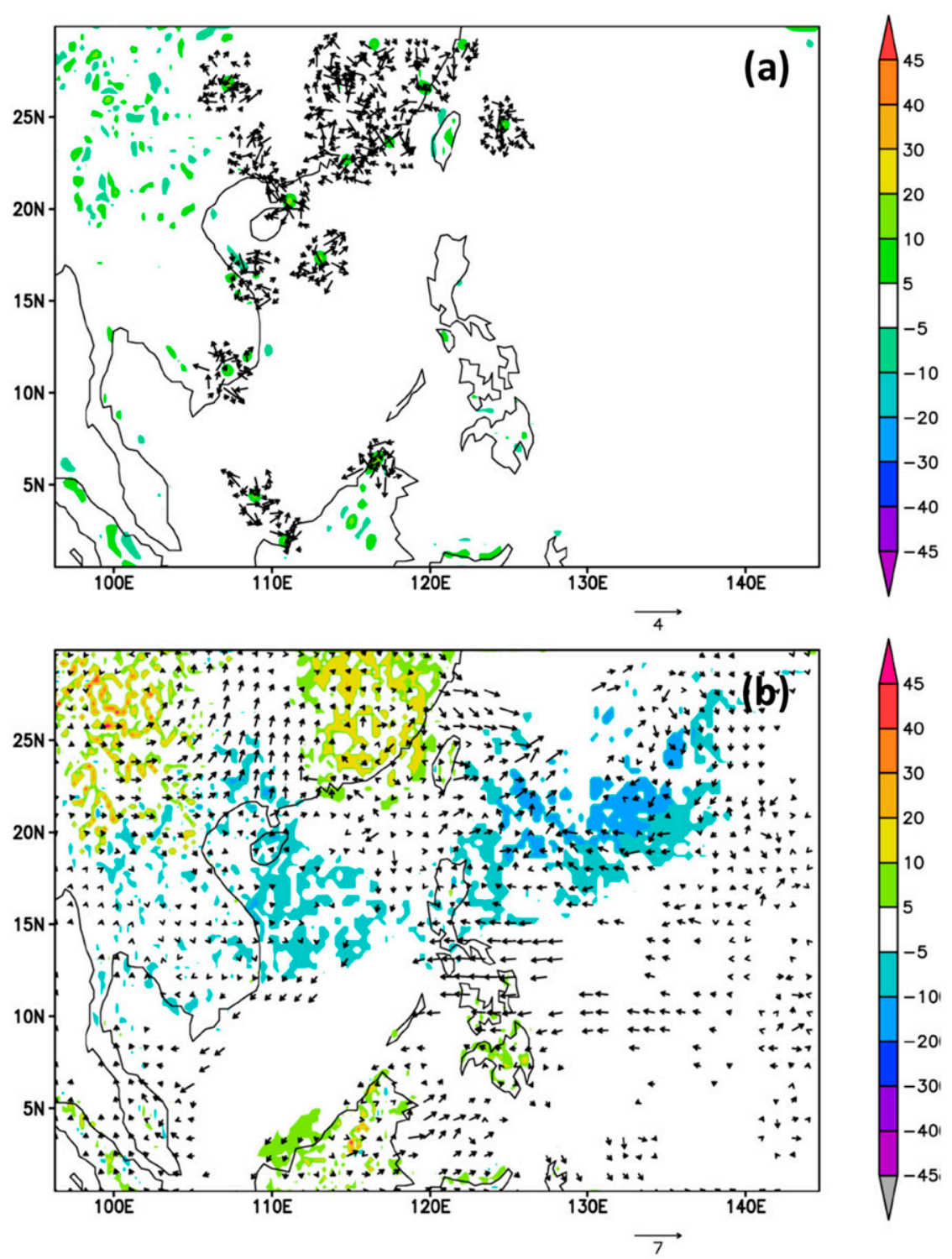

FIG. 6. As in Fig. 4c, but for (a) local station data only and (b) CIMSS AMV data only.

what the station data can. In particular, the enhancement of the monsoon trough over the DOIV is no longer seen after the AMV data are removed (cf. Figs. 6a,b). These results demonstrate the critical importance of the CIMSSAMV data in improving the GFS ambient environment that station data could not achieve alone. Such effects of the AMV data ultimately account for the differences in the forecast of Wutip's formation among different ensemble experiments as presented in the next sections.

\section{b. Prediction of Wutip's formation timing}

Given the improved initial conditions after assimilating augmented observations, ensemble forecasts from the WRF-LETKF system produce a range of possibilities for location and timing of Wutip's formation. We examine next in this subsection the prediction of the timing of Wutip's formation. Figure 7a shows the predictions of the formation time detected from the CTL ensemble forecasts for three consecutive cycles 1200 UTC 23 September, 0000 UTC 24 September, and 1200 UTC 24 September that correspond to 48-, 36-, and 24-h lead times prior to the observed formation of Wutip's depression. Unlike a general expectation that a shorter lead time would provide more reliable prediction, it is seen first from the CTL ensemble that the 36-h lead time forecasts provide the most consistent timing of Wutip's formation when verified against the actual formation time of Wutip at $\sim 1000$ UTC 25 September 

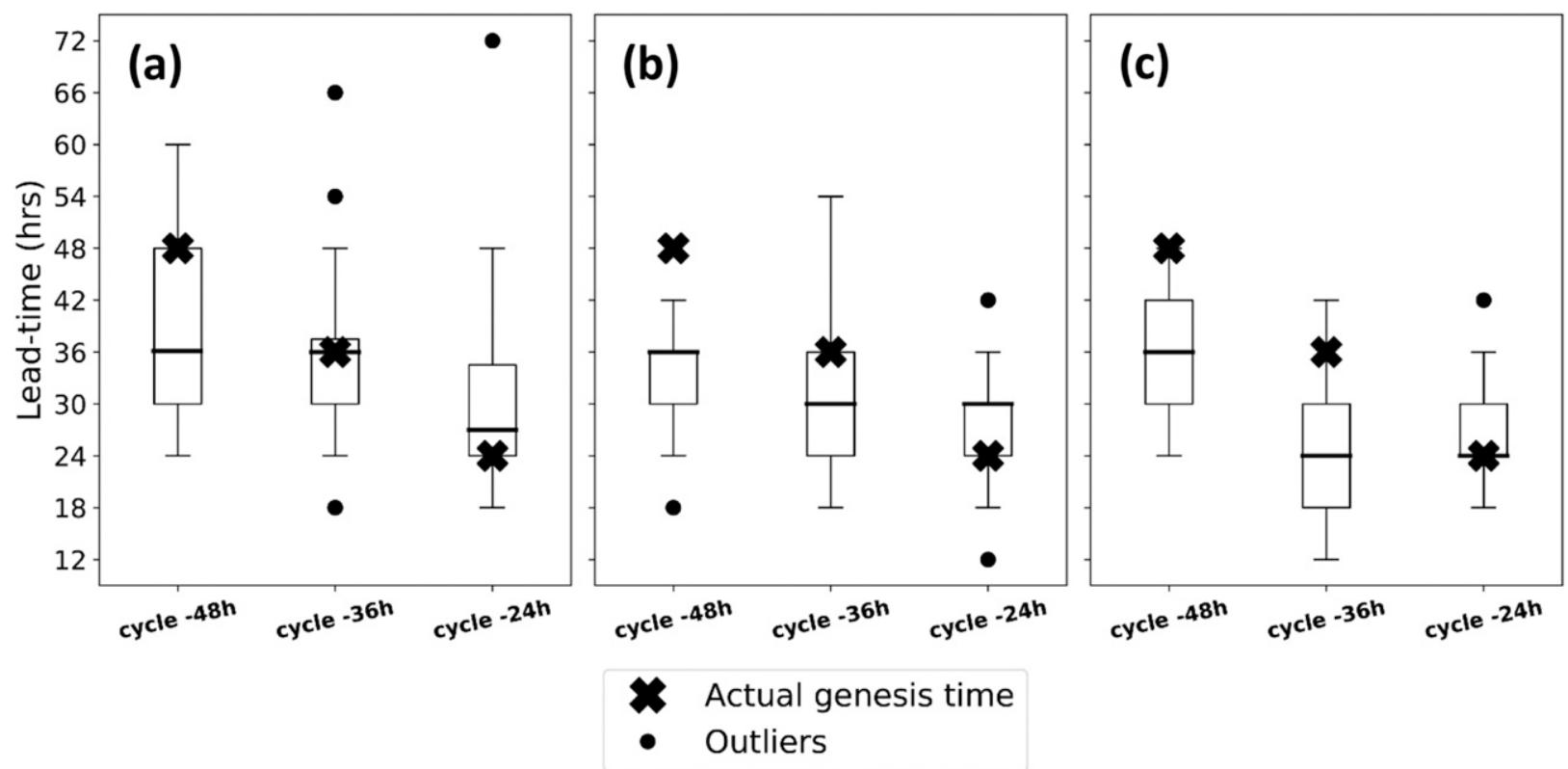

FIG. 7. Boxplots of the timing for Wutip formation for three consecutive cycles 1200 UTC 23 Sep, 0000 UTC 24 Sep, and 1200 UTC 24 Sep, which corresponds to 48, 36, and $24 \mathrm{~h}$ prior to the formation of Wutip depression for (a) CTL, (b) NAMV, and (c) NDA ensemble forecasts. The bold cross denotes the actual time that Wutip first became a tropical depression at 1200 UTC 25 Sep.

(Fig. 7a). The better formation timing forecasts at the 36-h lead time as compared to the 24-h lead time may appear somewhat surprising, because it is typically true that TC forecast skill decreases with lead times (e.g., Tallapragada et al. 2015, Tong et al. 2018). For TC formation forecasts, it turns out that this is not always the case, because the WRF Model would take some time to develop its coherent dynamics before a cyclogenesis can take place. Because Wutip's formation is strongly rooted in the monsoon trough (cf. Figs. 1 and 4), the 36-h interval appears to the right duration for the WRFLETKF system to capture good development of Wutip. A too close or too far lead time would lead to either insufficient time for the trough to spin up or the degradation of the WRF Model integration, even with augmented observation assimilation. This explains why the 48- and 24-h cycle could not provide good forecasts as compared to the 36-h lead time in the CTL ensemble.

To evaluate the impacts of the AMV data relative to station observations on the timing forecast of Wutip's formation, forecasts from sensitivity ensemble without the AMV data are provided in Fig. 7b. Consistent with the effects of the AMV data at the initial time (cf. Fig. 6a), the timing forecasts in these sensitivity experiments are immediately degraded, with incorrect timing of Wutip's formation as compared to the observed formation. Specifically, all NAMV ensemble forecasts exhibit earlier timing of formation at both 48- and 36-h lead times. As a result, the best ensemble forecast is now seen for 24-h cycle instead of 48 and 36-h cycles when the AMV data are removed. Additional comparison of the CTL and the NDA ensembles also shows that the formation timing forecasts without assimilation of any augmented observations are further degraded at 48- and 36-h lead times (Fig. 7c).

Similar to the experiment with no AMV assimilation, the NDA ensemble tends to develop a vortex earlier than the actual formation time for the 48- and 36-h cycles (Fig. 7c). Specifically for the 0000 UTC 24 September cycle (36-h lead time), the median of the timing forecast for Wutip's formation from the NDA ensemble is roughly $29-30 \mathrm{~h}$ into integration, which is $\sim 8 \mathrm{~h}$ earlier than the observed time of Wutip's formation. Such an earlier cyclogenesis process in the NDA ensemble turns out be due to the spin up of a wrong disturbance nearby as shown in the next section. The fact that the NDA experiment persistently develops a vortex within the same monsoon trough, albeit at a wrong time and location, confirms that the monsoon trough does play a critical role in Wutip's formation.

As an illustration of the difference in the large-scale environment among different lead times in the CTL ensemble, Fig. 8 shows the verification of the CTL ensemble forecasts against the NCEP final analysis (FNL) for the 48-, 36-, and 24-h cycles valid at 1200 UTC 25 September. Here, the verification is shown at the 850 -hPa level to best capture the monsoon trough characteristics. One notices a common feature in all three cycles is the underestimation 

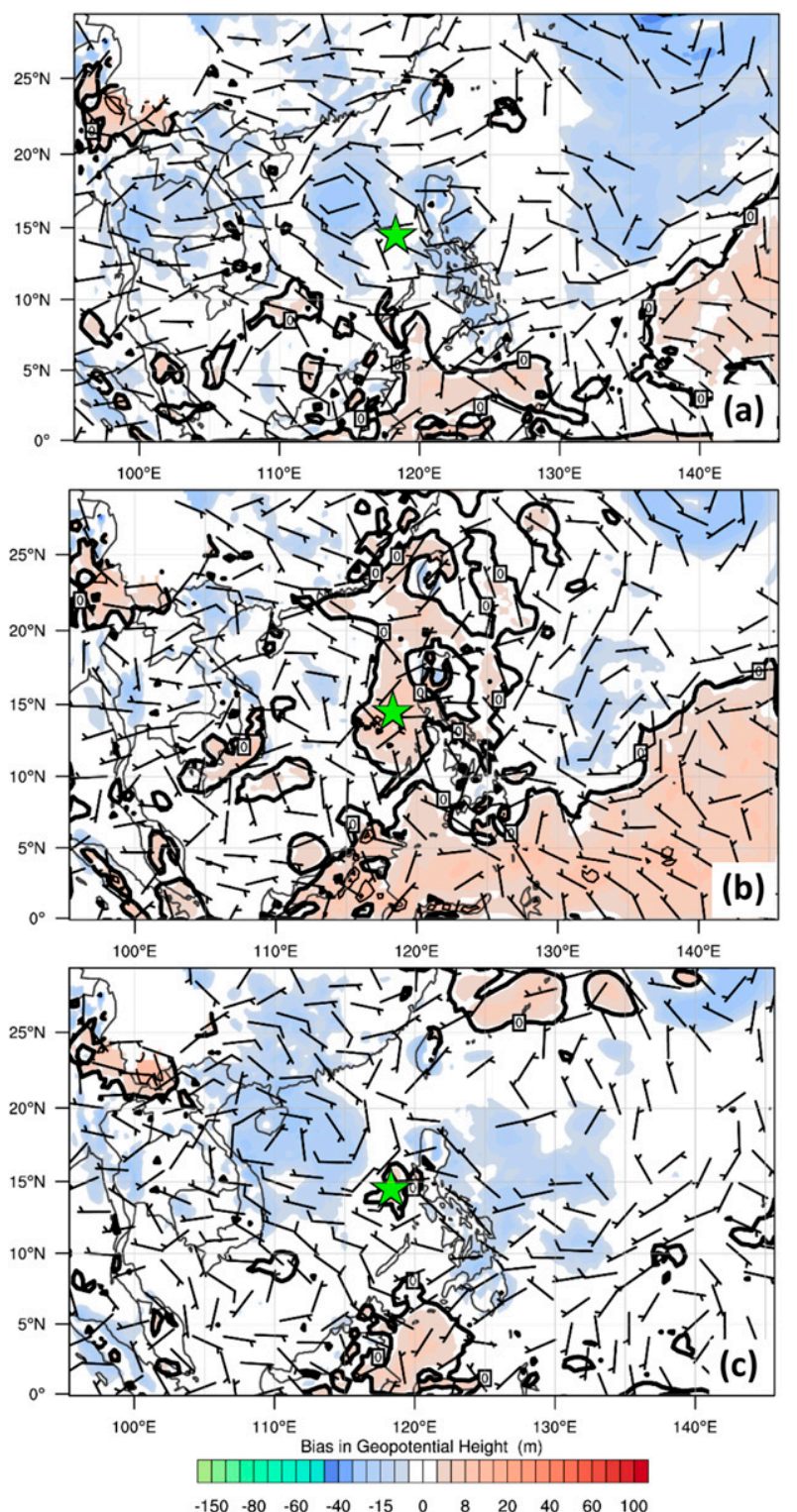

FIG. 8. Bias errors of the ensemble mean geopotential height perturbation (shaded; $\mathrm{m}$ ) and temperature (contours; K) and wind field (barbs) at $850 \mathrm{hPa}$ that are obtained from the CTL ensemble forecasts initialized at (a) 1200 UTC 23 Sep (48-h cycle), (b) 0000 UTC 24 Sep (36-h cycle), and (c) 1200 UTC 24 Sep (24-h cycle) against the NCEP final analysis valid at 1200 UTC 25 Sep. The star denotes the location where Wutip's depression was first reported.

of the monsoon strength, which is manifested as a negative geopotential height anomaly, along the trough in all three cycles. Although the 24-h cycle has the smallest overall bias distribution as expected from a shorter lead time, note that the 36-h cycle has the smallest negative bias along the trough among the three. In fact, the 36-h cycle is the only one that displays a broad lower pressure system (shown as a positive geopotential height anomaly relative to the FNL analysis) at the location where Wutip's formation occurred. Such emergence of a low pressure system is missing in the 48-h cycle as the monsoon strength is still weak at 1200 UTC 25 September, even after assimilating augmented observations. Likewise, the 24-h cycle could capture the right development and the timing, but the overall strength of the monsoon is still weak that the signal of Wutip's formation is not clear as shown in Fig. 8.

As forecast cycles are closer to the observed time of Wutip's formation, we notice that the timing forecast in the NDA ensemble fluctuates in a narrower range and tends to be closer to the observed time (Fig. 7c). In fact, the best timing forecast of Wutip's formation in the NDA ensemble is captured for the 24-h lead time cycle. Practically, such a better prediction of Wutip's formation timing for the cycle with a shorter lead time in the NDA ensemble as seen in Fig. 7 is anticipated. This is because the GFS initial condition at 1200 UTC 24 September is closer to the time when Wutip formed, and so the GFS initial analyses could better represent the cyclonic-rich environment along the monsoon trough where Wutip's precursor exists (cf. Figs. 4, 5, and 8). For earlier cycles, the GFS initial analyses could not fully capture the dynamics of the monsoon trough, and so it takes time for the WRF Model to spin up before the correct low pressure system can form. This result suggests that the good timing forecast in the NDA ensemble at the 24-h lead time is directly inherited from the GFS input data, rather than from the assimilation of augmented observation. Thus, the above result reiterates the importance of the AMV data in enhancing the initial strength of the monsoon trough for longer lead time forecasts of Wutip's formation.

\section{c. Prediction of Wutip's formation location}

Along with the forecasts of TC formation timing, predicting the location of TC formation is another important aspect of TC formation forecasts in practical applications. To examine this issue, Fig. 9 shows the distance errors of Wutip's formation location as forecast by the CTL ensemble for three cycles. Here, the distance errors are defined as a difference between a formation center detected from the model output and the actual location of Wutip's tropical depression stage first recorded in the best track dataset. On average, the CTL formation location forecast has an error of $\sim 300 \mathrm{~km}$, with the largest errors for the $48-\mathrm{h}$ cycle $(\sim 380 \mathrm{~km})$. Consistent with the best timing forecast at the 36-h lead time, we notice that the $36-\mathrm{h}$ cycle also has the smaller distance errors for the formation location forecast as compared to the NDA ensemble. In contrast, removing 


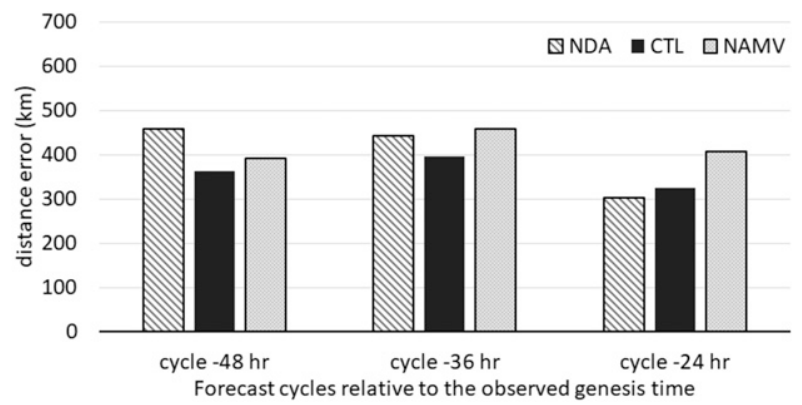

FIG. 9. Ensemble mean distance errors between the center of Wutip's formation obtained from the model forecast against the observed location of the tropical depression reported at 1200 UTC 25 Sep for three cycles of 48, 36, and $24 \mathrm{~h}$ obtained from the CTL forecast (black), NDA forecast (stripe), and NAMV forecasts (light shaded).

AMV data in the NAMV ensemble results in large distance errors, especially for the 36-h cycle as seen in Fig. 9.

Such a larger error in the location of Wutip's formation in the NDA and NAMV ensembles at the 48-h lead time turns out to be a consequence of an incorrect spin up of a nearby tropical disturbance very early in several ensemble (Fig. 10). This explains why the average location errors in the NAMV ensemble are larger by $\sim 25 \%$ as compared to those in the CTL ensemble. The larger location errors are most apparent for the 36-h cycle, as both the NAMV and NDA ensembles could not capture the correct timing of Wutip's formation. As a result, the large-scale environment in the NDA ensemble changes dramatically, leading to a location of Wutip's formation shifted too far to the west of the actual location. Similar to the timing forecast, one notices again that the NDA ensemble has the smallest location forecast errors for the 24-h cycle, despite no augmented observation. This is because the GFS initial condition is much improved about $24 \mathrm{~h}$ prior to the formation of Wutip (cf. Fig. 5) that the impacts of local augmented observations are no longer significant at the large scale.

It should be mentioned that the outperformance of the CTL ensemble in forecasting Wutip's formation location comes from two different factors including 1) improved initial conditions after assimilating augmented observation, and 2) the multiple-physics ensemble. While the benefit of improved initial conditions is best seen at 24-36-h lead time, the use of multiple physics ensemble plays more important role at the longer lead time in our WRF-LETKF system (see Kieu et al. 2014; Du et al. 2017). Specifically, a multiplephysics ensemble helps develop a better background covariance matrix such that observational information can be optimally assimilated when cycling with time. In
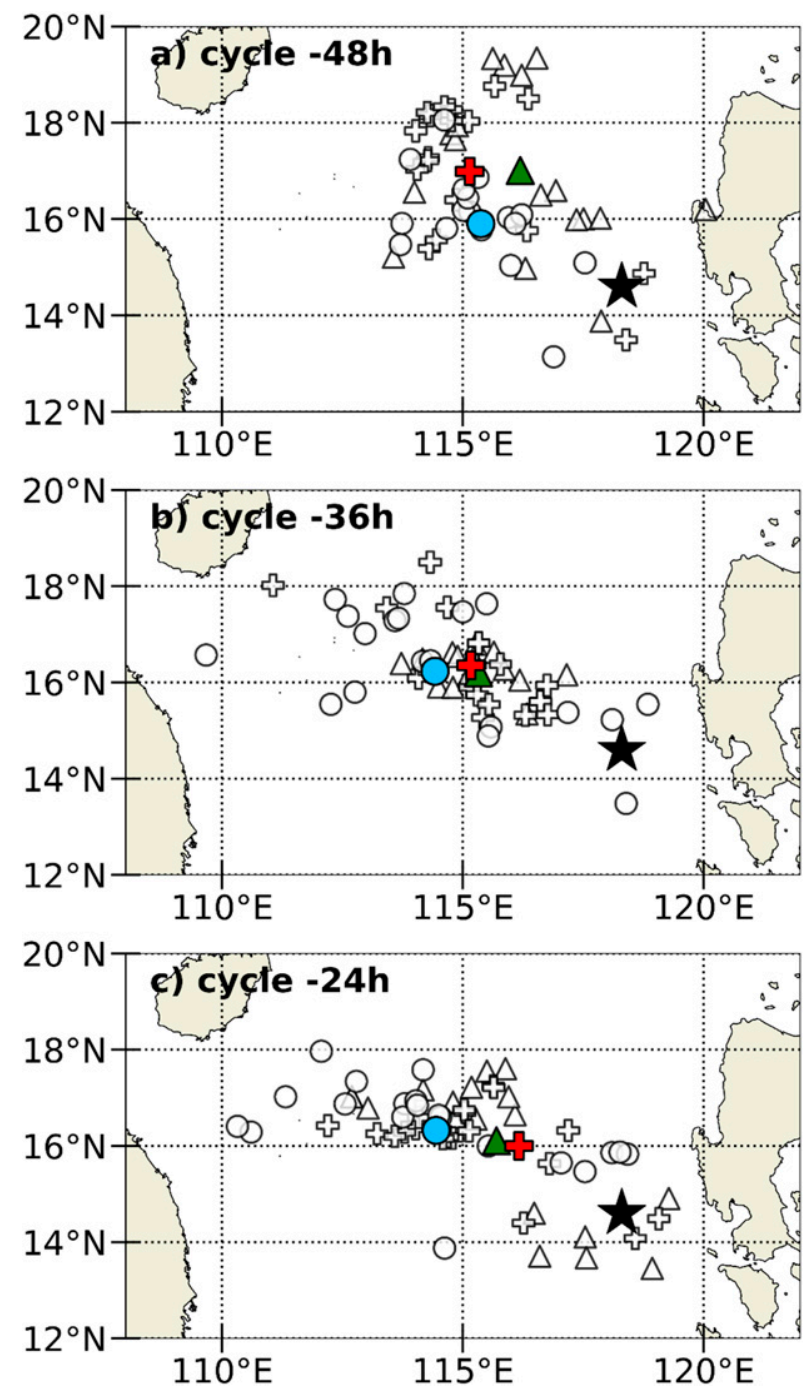

FIG. 10. Distribution of the location of the Wutip's formation centers as forecast by the CTL ensemble (triangle), the NAMV ensemble (circle), and NDA ensemble (cross) for (a) 48-, (b) 36-, and (c) 24-h cycles. Color symbols denote the ensemble means of corresponding forecasts.

the case of Wutip's formation, the benefit of different physical parameterization schemes in the CTL experiment is best seen in terms of the spread of the ensemble in predicting the location of Wutip's formation. As shown in Fig. 11, about $80 \%$ of ensemble members could capture Wutip's formation in all experiments observations or not. Note that the forecast of Wutip's formation location is very diverse among different ensemble members, yet it is still distributed along the monsoon trough in the DOIV as seen in Fig. 10. This clustering of Wutip's formation centers along the monsoon trough highlights the favorable environment of the trough for TC development.

We note also from Fig. 11 that several ensemble members appear to have very consistent performance in 

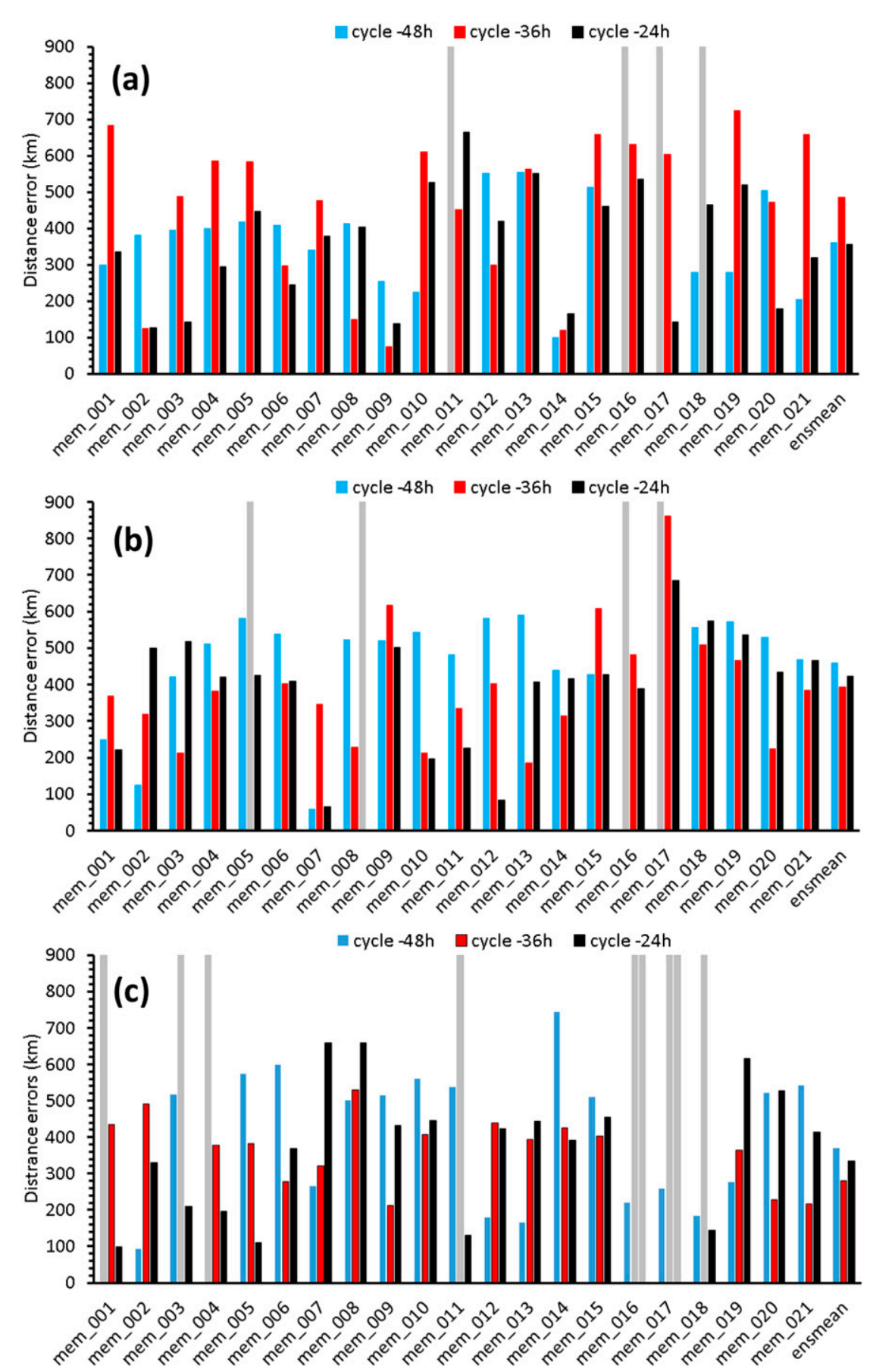

FIG. 11. Individual member distance errors between the center of Wutip's formation obtained from the model forecast against the observed location of the tropical depression reported at 1200 UTC 25 Sep for three different cycles $48 \mathrm{~h}$ (blue), 36 h (red), and $24 \mathrm{~h}$ (black) for (a) CTL forecast, (b) NDA forecast, and (c) NAMV forecasts. Gray columns denote nondeveloping members. The abbreviation mem_00i denotes the $i$ th member of the each ensemble.

terms of the location of Wutip's formation for all cycles. Recall that each ensemble member in the WRF-LETKF system, by design, has its own set of physical parameterization schemes. Thus, the consistently worse performance of few ensembles may indicate an improper combination of model physics that are not suitable for the cyclogenesis process associated with monsoon troughs (combinations 16, 17, and 18 in Table 1. See also Fig. 11). 
(a) 48-hr dev

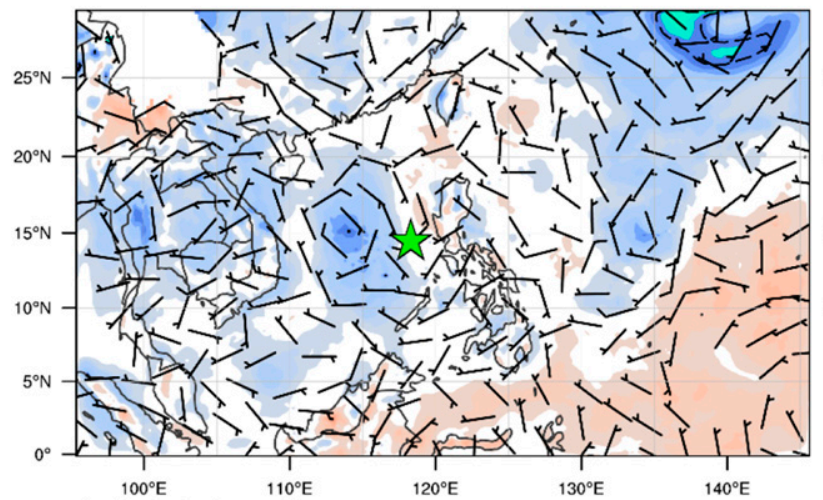

(c) $36-\mathrm{hr} \mathrm{dev}$

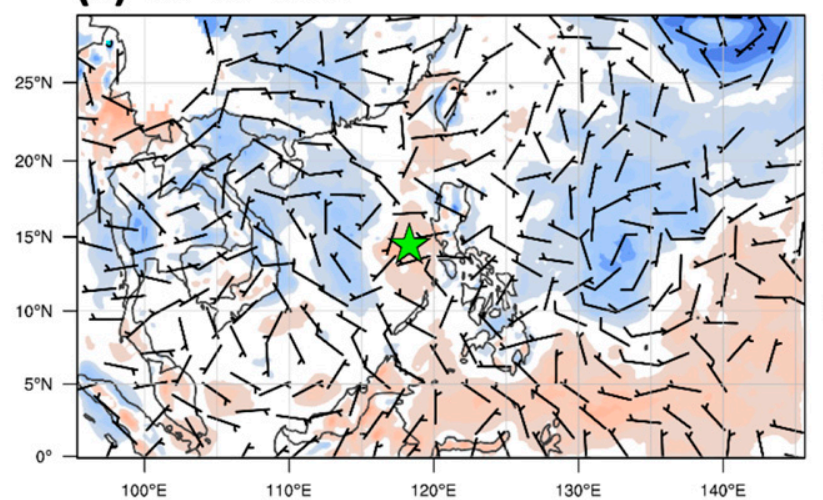

(b) 48-hr nondev

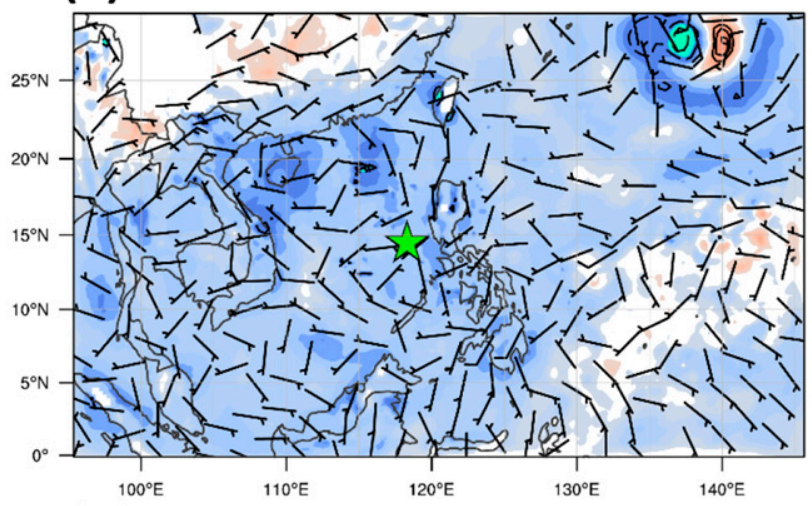

(d) 36-hr nondev

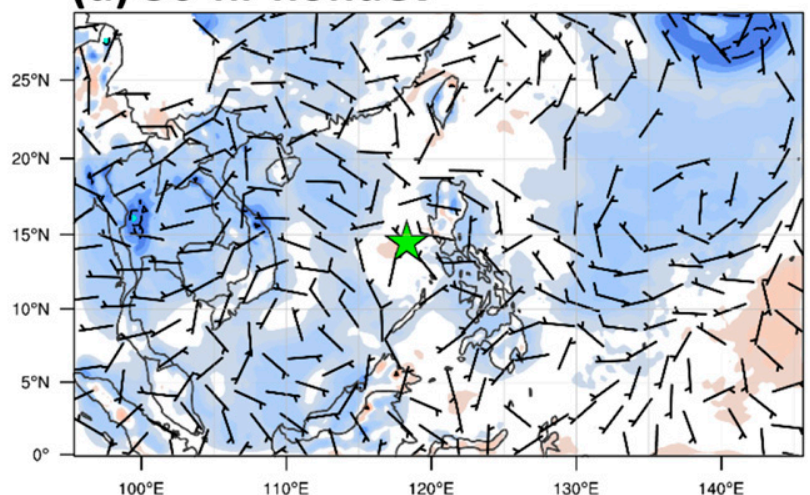

Bias in Geopotential Height $(\mathrm{m})$

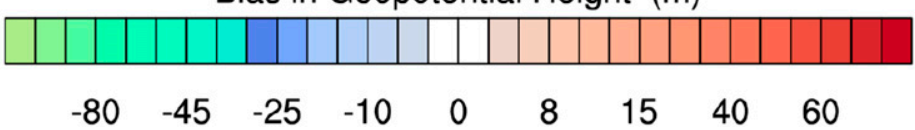

FIG. 12. As in Fig. 8, but for the (left) developing members and (right) nondeveloping members from the CTL forecast for (a),(b) 48- and (c),(d) 36-h forecast cycles.

As an illustration, Fig. 12 compares the large-scale bias for two groups of CTL members initialized at 1200 UTC 23 September that are verified against the FNL analyses at 1200 UTC 25 September. One group include all members in the CTL ensemble that could exhibit Wutip's formation around 1200 UTC 25 September (referred to as a developing group), and the other group composing of the rest of the CTL ensemble members that do not capture any development of Wutip (hereafter a nondeveloping group). As shown in Fig. 12, all nondeveloping members show a broad negative geopotential height bias over the entire domain, especially in the DOIV area where Wutip develops. In contrasts, the developing members display a less negative geopotential height bias, thus indicating a better evolution of the monsoon trough required to Wutip's formation despite similar enhanced initial condition after assimilating augmented observations. In this regard, a proper choice of physical parameterization is of importance for TC formation forecast that an ensemble with a single set of model physics may not fully capture.

To see the large-scale structure of the two groups beyond the verification against the FNL analyses, Fig. 13 shows the composite horizontal distributions of water mixing ratio and geopotential perturbation for the developing and the nondeveloping groups similar to analyses in Fig. 8. In general, both geopotential height and wind fields display a broad similarity between the two groups, with similar appearance of a monsoon trough and a low pressure system associated with Typhoon Usagi nearby. Despite these similar large-scale features, there is a significant difference between these two groups in terms of the strength of the monsoon trough and related distribution of moisture field (cf. also Fig. 8). That is, the low pressure band along the trough in the developing group has a more distinct elongated structure that concentrates in a narrow zone from Vietnam Central Highland to the Philippine Sea. Corresponding 

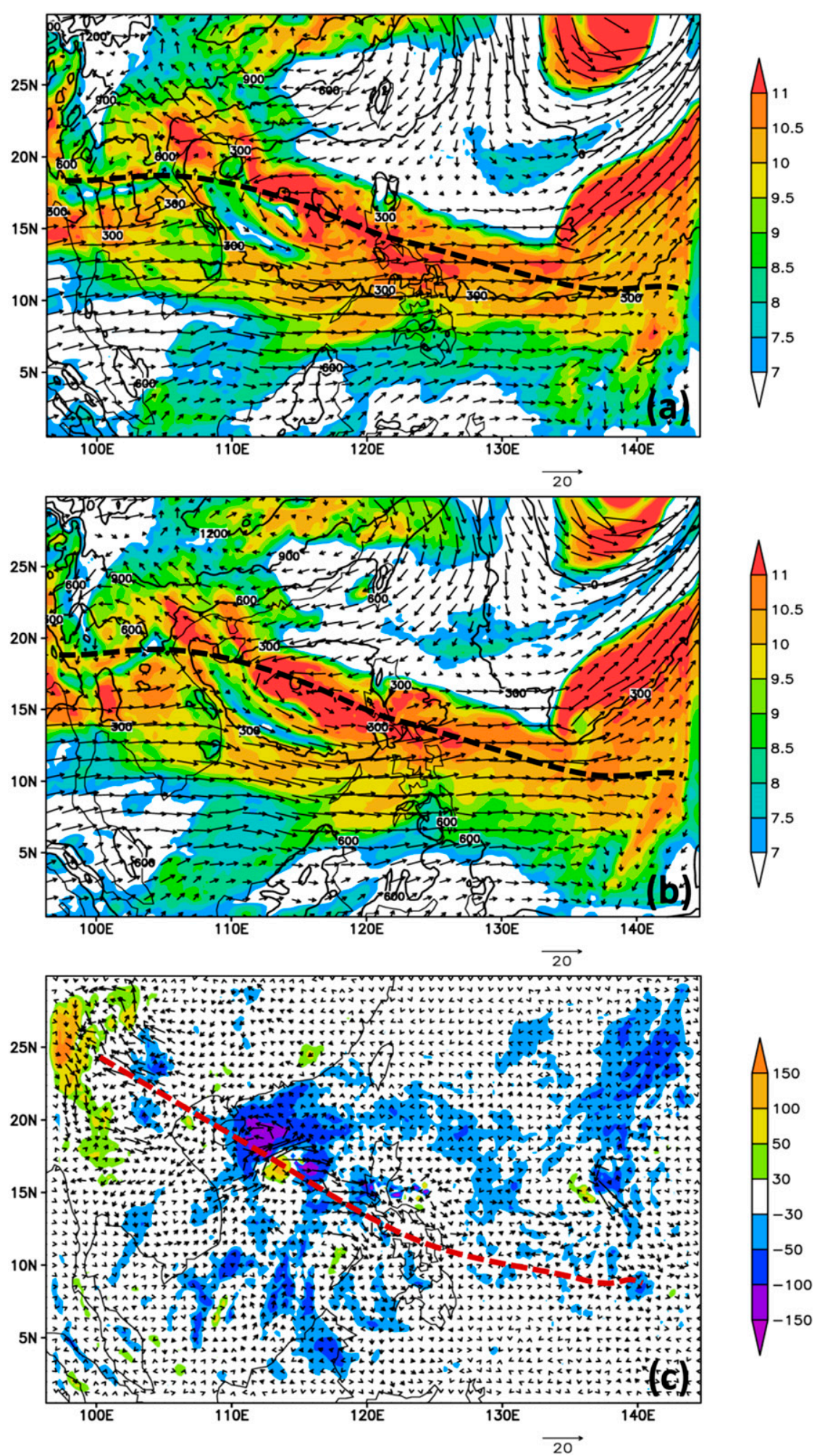

FIG. 13. Horizontal distributions of the mixing ratio (shaded; $10^{-3} \mathrm{~kg} \mathrm{~kg}^{-1}$ ) and the geopotential perturbation (contours; $\mathrm{m}^{2} \mathrm{~s}^{-2}$ ) at 800 -hPa level valid at 1200 UTC 25 Sep when Wutip first became a depression (48-h lead time for the cycle initialized at 1200 UTC 23 Sep 2013) that are averaged for (a) a group of developing members, (b) a group of nondeveloping members in the CTL ensemble, and (c) the difference of between the two groups. The dashed black lines in (a) and (b) denote the location of monsoon trough, and the red line in (c) denotes the band where the difference in surface convergence is most significant. 
to this stronger low pressure anomaly is a larger lowlevel converging wind and higher water content along the trough in the developing group. Such a larger lowlevel convergence of moist air results in an enhanced convective activity along the trough, thus producing a low-level warm core and deepening the surface pressure prior to the formation of Wutip, which is absent in the nondeveloping group.

These differences provide some evidence that the cyclogenesis can proceed from a tropical disturbance embedded within the preexisting low pressure band as seen in the developing group. Of course, these results still cannot explain the physical mechanism by which the CTL ensemble could develop such a difference between the two groups, because any physical mechanism would require cause-and-effect analyses beyond diagnostic analyses. However, the above results could at least reveal the requirement for Wutip's formation that the WRF-LETKF system could capture after assimilating augmented observations. We should emphasize that these results do not imply that the same physical combinations will work well for all TC formation cases with different large-scale environment. Nonetheless, the results seen in Figs. 11-13 could at least confirm that the use of multiple physical options for the ensemble forecast is suitable for real-time forecasts of TC formation for which a single set of physical parameterization schemes could not achieve.

\section{Conclusions}

In this study, the sensitivity of TC formation forecast to different types of augmented observations was examined using the local ensemble transform Kalman filter (LETKF) algorithm. By choosing a case of Typhoon Wutip (2013) that formed within a typical monsoon trough in the WPAC basin, it was demonstrated that the initial representation of a large-scale monsoon trough, which hosted the formation of Typhoon Wutip, is sensitive to augmented observations. Specifically, a direct initialization of the WRF Model from the global GFS analyses at 48 and $36 \mathrm{~h}$ prior to Wutip's formation displayed a weak strength of the monsoon trough. Assimilation of augmented observations including satellite atmospheric motion wind vectors (AMV), local stations, and surface data could improve the initial monsoon trough representation by allowing for stronger low-level convergence toward the trough, thus generating a more favorable environment prone to cyclogenesis.

Further separating the local stations/surface data and the CIMSS-AMV demonstrated that the satellite AMV data plays a more essential role in enhancing the monsoon strength. Despite its data distribution confined mostly to mid- to upper levels, the much larger coverage of the AMV data results in a broader improvement of environmental flows associated with the entire monsoon trough. While the AMV data are in principle included in the global GFS analyses, reassimilating this data at a higher resolution of a regional model can capture some additional benefit that simple interpolation from the global coarse resolution to the regional higher resolution could not achieve. As the WRF-LETKF system is cycled, the cross correlation of the LETKF background covariance matrix could spread the assimilated information over the entire domain, thus increasing the impacts of augmented observations on the model initial conditions.

Given the improvement in the initial large-scale environment after assimilating augmented observations, ensemble forecasts of Wutip's formation showed a range of outcomes in terms of the timing and the location of Wutip's formation. For the timing forecast, the CTL ensemble in which all augmented observations were assimilated showed the most benefit for the cycle at $36 \mathrm{~h}$ prior to Wutip's formation. For the cycle that is sufficiently close to the actual time when Wutip formed $(<24 \mathrm{~h})$, the skill of the formation timing forecast by the CTL ensemble is comparable to the forecasts that are directly initialized from the GFS input data without any assimilation (NDA). Likewise, the CTL forecast cycle $48 \mathrm{~h}$ prior to the observed formation of Wutip could not properly predict the timing of Wutip's formation, regardless of assimilating augmented observations or not. Thus, TC formation associated with monsoon troughs appears to possess limited predictability that improving initial conditions may not lengthen the forecast lead time. On the one hand, this result indicates that the GFS initial condition could not properly capture the strength of the monsoon trough needed for cyclogenesis to occur. Therefore, assimilating more local observations can help improve the GFS initial condition and increase the chance of predicting tropical cyclogenesis associated with monsoon troughs at the 36 -h lead time. On the other hand, this result also suggests that the GFS initial condition is generally sufficient for TC formation forecast at shorter lead time, even in the absence of any local augmented observations.

Similar impacts of augmented observation on the forecast of Wutip's formation location were also documented. For the CTL ensemble, the location where Wutip formed was best captured at the 36 -h lead time as compared to other lead times. At the shorter 24-h or longer 48-h lead time, there is no clear benefit of assimilating augmented observations. The most notable feature in the forecast of TC formation location without 
assimilating augmented observations is that the model tended to spin up different tropical disturbances that were close to Vietnam's coastal region for the 48- and 36-h cycles. Such incorrect development of nearby disturbances in the NDA ensemble resulted in a large distance error in Wutip's formation location forecast as compared to the CTL ensemble. Additional experiments in which only station and surface observations were used in assimilation cycles showed a similar degraded skill as in the NDA forecasts. These results reveal the critical role of the AMV data in improving Wutip's formation forecast at the 36-h lead time.

It should be cautioned that the results obtained in this study are specific to the case of Typhoon Wutip whose formation was associated with a monsoonal system. These results are therefore not representative and may not be applied to other tropical cyclogenesis pathways, nor could they reveal the general physical mechanisms of TC formation in the WPAC basin. However, our results could present the importance of augmented observations, especially the satellite AMV data, for the prediction of TC formation at certain lead times that are vital for operational TC forecasts. In this regard, this study highlights the need of augmented observations for prediction of TC formation that one has to properly examine when designing future observing systems.

Acknowledgments. This research was supported by the Vietnam National Foundation for Science and Technology (KC09.12/16-20). We thank three anonymous reviewers for their very constructive comments and suggestions that have helped improved this work substantially.

Data availability statement: All local and augmented observational data used in this study are available for public release upon request. The GFS forecast data used to initialize the WRF Model in this study are provided directly by the NOAA/NCEP data portal, which is also freely accessible.

\section{REFERENCES}

Aberson, S. D., A. Aksoy, K. J. Sellwood, T. Vukicevic, and X. Zhang, 2015: Assimilation of high-resolution tropical cyclone observations with an ensemble Kalman filter using HEDAS: Evaluation of 2008-2011 HWRF forecasts. Mon. Wea. Rev., 143, 511-523, https://doi.org/10.1175/MWR-D-1400138.1.

Avila, L. A., and R. J. Pasch, 1992: Atlantic tropical systems of 1991. Mon. Wea. Rev., 120, 2688-2696, https://doi.org/10.1175/ 1520-0493(1992)120<2688:ATSO > 2.0.CO;2.

Bister, M., and K. A. Emanuel, 1997: The genesis of Hurricane Guillermo: TEXMEX analyses and a modeling study. Mon. Wea. Rev., 125, 2662-2682, https://doi.org/10.1175/1520-0493(1997) $125<2662$ :TGOHGT $>2.0 . \mathrm{CO} ; 2$.
Carr, L. E., and R. L. Elsberry, 1995: Monsoonal interactions leading to sudden tropical cyclone track changes. Mon. Wea. Rev., 123, 265-290, https://doi.org/10.1175/1520-0493(1995) $123<0265$ :MILTST>2.0.CO;2.

Chan, J. C. L., and R. H. Kwok, 1999: Tropical cyclone genesis in a global numerical weather prediction model. Mon. Wea. Rev., 127, 611-624, https://doi.org/10.1175/1520-0493(1999) 127<0611:TCGIAG $>2.0 . \mathrm{CO} ; 2$.

— , and M. Xu, 2009: Inter-annual and inter-decadal variations of landfalling tropical cyclones in East Asia. Part I: Time series analysis. Int. J. Climatol., 29, 1285-1293, https:// doi.org/10.1002/joc.1782.

Chen, J., S. Lin, L. Zhou, X. Chen, S. Rees, M. Bender, and M. Morin, 2019: Evaluation of tropical cyclone forecasts in the next generation global prediction system. Mon. Wea. Rev., 147, 3409-3428, https://doi.org/10.1175/MWR-D-18-0227.1.

Cheung, K. K., and R. L. Elsberry, 2002: Tropical cyclone formations over the western North Pacific in the Navy Operational Global Atmospheric Prediction System forecasts. Wea. Forecasting, 17, 800-820, https://doi.org/10.1175/ 1520-0434(2002)017<0800:TCFOTW >2.0.CO;2.

Choi, J.-W., Y. Cha and J.-Y. Kim, 2017: Change in the tropical cyclone activity around Korea by the East Asian summer monsoon. Geosci. Lett., 4, 2, https://doi.org/10.1186/S40562017-0067-6.

Cinco, T. A., and Coauthors, 2016: Observed trends and impacts of tropical cyclones in the Philippines. Int. J. Climatol., 36, 4638-4650, https://doi.org/10.1002/joc.4659.

DeMaria, M., 1996: The effect of vertical shear on tropical cyclone intensity change. J. Atmos. Sci., 53, 2076-2088, https://doi.org/ 10.1175/1520-0469(1996)053<2076:TEOVSO > 2.0.CO;2.

Dickinson, M., and J. Molinari, 2002: Mixed Rossby-gravity waves and western Pacific tropical cyclogenesis. Part I: Synoptic evolution. J. Atmos. Sci., 59, 2183-2196, https://doi.org/10.1175/ 1520-0469(2002)059<2183:MRGWAW >2.0.CO;2.

Du, T. D., T. Ngo-Duc, and C. Kieu, 2017: Initializing the WRF Model with tropical cyclone real-time reports using the ensemble Kalman filter algorithm. Pure Appl. Geophys., 174, 2803-2825, https://doi.org/10.1007/s00024-017-1568-0.

Dunkerton, T. J., M. T. Montgomery, and Z. Wang, 2009: Tropical cyclogenesis in a tropical wave critical layer: Easterly waves. Atmos. Chem. Phys., 9, 5587-5646, https://doi.org/10.5194/ acp-9-5587-2009.

Frank, W. M., and P. E. Roundy, 2006: The role of tropical waves in tropical cyclogenesis. Mon. Wea. Rev., 134, 2397-2417, https:// doi.org/10.1175/MWR3204.1.

Gray, W. M., 1968: Global view of the origin of tropical disturbances and storms. Mon. Wea. Rev., 96, 669-700, https://doi.org/10.1175/ 1520-0493(1968)096<0669:GVOTOO > 2.0.CO;2.

_ 1982: Tropical cyclone genesis and intensification. Tropics in Atmospheric and Oceanographic Sciences: Intense Atmospheric Vortices, L. Bengtsson and J. Lighthill, Eds., SpringerVerlag, 3-20.

Halperin, D. J., H. E. Fuelberg, R. E. Hart, J. H. Cossuth, P. Sura, and R. J. Pasch, 2013: An evaluation of tropical cyclone genesis forecasts from global numerical models. Wea. Forecasting, 28, 1423-1445, https://doi.org/10.1175/WAF-D-13-00008.1. , and -2016 : Verification of tropical cyclone genesis forecasts from global numerical models: Comparisons between the North Atlantic and eastern North Pacific basins. Wea. Forecasting, 31, 947-955, https://doi.org/10.1175/WAF-D-15-0157.1.

Halverson, J., and Coauthors, 2007: NASA's tropical cloud systems and processes experiment: Investigating tropical cyclogenesis 
and hurricane intensity change. Bull. Amer. Meteor. Soc., 88, 867-882, https://doi.org/10.1175/BAMS-88-6-867.

Harr, P. A., R. L. Elsberry, and J. C. L. Chan, 1996: Transformation of a large monsoon depression to a tropical storm during TCM-93. Mon. Wea. Rev., 124, 2625-2643, https://doi.org/ 10.1175/1520-0493(1996)124<2625:TOALMD>2.0.CO;2.

Holmlund, K., C. Velden, and M. Rohn, 2001: Enhanced automated quality control applied to high-density satellite-derived winds. Mon. Wea. Rev., 129, 517-529, https://doi.org/10.1175/ 1520-0493(2001)129<0517:EAQCAT>2.0.CO;2.

Holt, C., I. Szunyogh, G. Gyarmati, S. M. Leidner, and R. N. Hoffman, 2015: Assimilation of tropical cyclone observations: Improving the assimilation of TCVitals, scatterometer winds, and dropwindsonde observations. Mon. Wea. Rev., 143, 39563980, https://doi.org/10.1175/MWR-D-14-00158.1.

Hunt, B. R., E. J. Kostelich, and I. Szunyogh, 2007: Efficient data assimilation for spatiotemporal chaos: A local ensemble transform Kalman filter. Physica D, 230, 112-126, https:// doi.org/10.1016/j.physd.2006.11.008.

Kang, J.-S., E. Kalnay, J. Liu, I. Fung, T. Miyoshi, and K. Ide, 2011: "Variable localization" in an ensemble Kalman filter: Application to the carbon cycle data assimilation. J. Geophys. Res., 116, D09110, https://doi.org/10.1029/2010JD014673.

Kieu, C. Q., and D.-L. Zhang, 2008: Genesis of Tropical Storm Eugene (2005) from merging vortices associated with ITCZ breakdowns. Part I: Observational and modeling analyses. J. Atmos. Sci., 65, 3419-3439, https://doi.org/ 10.1175/2008JAS2605.1.

$\longrightarrow$, and — 2009: Genesis of Tropical Storm Eugene (2005) from merging vortices associated with ITCZ breakdowns. Part II: Roles of vortex merger and ambient potential vorticity. J. Atmos. Sci., 66, 1980-1996, https://doi.org/10.1175/2008JAS2905.1.

, M. T. Nguyen, T. M. Hoang, and T. Ngo-Duc, 2012: Sensitivity of the track and intensity forecasts of Typhoon Megi (2010) to satellite-derived atmospheric motion vectors with the ensemble Kalman filter. J. Atmos. Oceanic Technol., 29, 1794-1810, https://doi.org/10.1175/JTECH-D-12-00020.1.

_, P. T. Minh, and H. T. Mai, 2014: An application of the multiphysics ensemble Kalman filter to typhoon forecast. Pure Appl. Geophys., 171, 1473-1497, https://doi.org/10.1007/S00024-0130681-Y.

Lander, M. A., and G. J. Holland, 1993: On the interaction of tropical cyclone-scale vortices. I. Observations. Quart. J. Roy. Meteor. Soc., 119, 1347-1361, https://doi.org/10.1002/qj.49711951406.

- 1994: Description of a monsoon gyre and its effects on the tropical cyclones in the western North Pacific during August 1991. Wea. Forecasting, 9, 640-654, https://doi.org/10.1175/ 1520-0434(1994)009<0640:DOAMGA>2.0.CO;2.

Le Marshall, J., A. Rea, L. Leslie, R. Seecamp, and M. Dunn, 2004: Error characterisation of atmospheric motion vectors. Aust. Meteor. Mag., 53, 123-131.

Li, J., J. Li, C. Velden, P. Wang, T. J. Schmit, and J. Sippel, 2020: Impact of rapid-scan-based dynamical information from GOES16 on HWRF hurricane forecasts. J. Geophys. Res. Atmos., 125, e2019JD031647, https://doi.org/10.1029/2019JD031647.

Li, J. P., and Q. C. Zeng, 2002: A unified monsoon index. Geophys. Res. Lett., 29, 1274, https://doi.org/10.1029/2001GL013874.

$\longrightarrow$, and - 2003: A new monsoon index and the geographical distribution of the global monsoons. Adv. Atmos. Sci., 20, 299302, https://doi.org/10.1007/s00376-003-0016-5.

Lin, I.-I., and J. C. Chan, 2015: Recent decrease in typhoon destructive potential and global warming implications. Nat. Commun., 6, 7182, https://doi.org/10.1038/ncomms8182.
Miyoshi, T., and M. Kunii, 2012: The local ensemble transform Kalman filter with the weather research and forecasting model: Experiments with real observations. Pure Appl. Geophys., 169, 321-333, https://doi.org/10.1007/s00024-011-0373-4.

Oouchi, K., J. Yoshimura, H. Yoshimura, R. Mizuta, S. Kusunoki, and A. Noda, 2006: Tropical cyclone climatology in a globalwarming climate as simulated in a $20 \mathrm{~km}$-mesh global atmospheric model: Frequency and wind intensity analyses. J. Meteor. Soc. Japan, 84, 259-276, https://doi.org/10.2151/ jmsj.84.259.

Ott, E., and Coauthors, 2004: A local ensemble Kalman filter for atmospheric data assimilation. Tellus, 56A, 415-428, https:// doi.org/10.3402/tellusa.v56i5.14462.

$\mathrm{Pu}, \mathrm{Z}$., S. Zhang, M. Tong, and V. Tallapragada, 2016: Influence of the self-consistent regional ensemble background error covariance on hurricane inner-core data assimilation with the GSI-based hybrid system for HWRF. J. Atmos. Sci., 73, 4911-4925, https://doi.org/10.1175/JAS-D-16-0017.1.

Simpson, J., E. A. Ritchie, G. J. Holland, J. Halverson, and S. Stewart, 1997: Mesoscale interactions in tropical cyclone genesis. Mon. Wea. Rev., 125, 2643-2661, https://doi.org/10.1175/ 1520-0493(1997)125<2643:MIITCG>2.0.CO;2.

Skamarock, W. C., J. B. Klemp, J. Dudhia, D. O. Gill, D. M. Barker, W. Wang, and J. G. Powers, 2005: A description of the Advanced Research WRF version 2. NCAR Tech. Note NCAR/TN468+STR, 88 pp., https://doi.org/10.5065/D6DZ069T.

Snyder, C., and F. Zhang, 2003: Assimilation of simulated Doppler radar observations with an ensemble Kalman filter. Mon. Wea. Rev., 131, 1663-1677, https://doi.org/10.1175//2555.1.

Strachan, J., P. L. Vidale, K. Hodges, M. Roberts, and M.-E. Demory, 2013: Investigating global tropical cyclone activity with a hierarchy of AGCMs: The role of model resolution. J. Climate, 26, 133-152, https://doi.org/10.1175/JCLI-D-1200012.1.

Szunyogh, I., E. J. Kostelich, G. Gyarmati, E. Kalnay, B. R. Hunt, E. Ott, E. Satterfield, and J. A. Yorke, 2008: A local ensemble transform Kalman filter data assimilation system for the NCEP global model. Tellus, 60A, 113-130, https://doi.org/ 10.1111/j.1600-0870.2007.00274.X.

Tallapragada, V., C. Q. Kieu, Y. Kwon, Q. Liu, S. Trahan, Z. Zhang, M. Tong, and B. Strahl, 2015: Forecasting tropical cyclones in the western North Pacific basin using the NCEP operational HWRF: Real-time implementation in 2012. Wea. Forecasting, 30, 1355-1373, https://doi.org/10.1175/WAF-D14-00138.1.

Tan, W., X. Wang, W. Wang, C. Wang, and J. Zuo, 2016: Different responses of sea surface temperature in the South China Sea to various El Niño events during boreal autumn. J. Climate, 29, 1127-1142, https://doi.org/10.1175/JCLI-D-15-0338.1.

Tong, M., and Coauthors, 2018: Impact of assimilating aircraft reconnaissance observations on tropical cyclone initialization and prediction using operational HWRF and GSI ensemblevariational hybrid data assimilation. Mon. Wea. Rev., 146, 4155-4177, https://doi.org/10.1175/MWR-D-17-0380.1.

Velden, C. S., C. M. Hayden, W. P. Menzel, J. L. Franklin, and J. S. Lynch, 1992: The impact of satellite-derived winds on numerical hurricane track forecasting. Wea. Forecasting, 7, 107-118, https:// doi.org/10.1175/1520-0434(1992)007<0107:TIOSDW>2.0.CO;2.

— C. Hayden, S. Nieman, W. Menzel, S. Wanzong, and J. Goerss, 1997: Upper-tropospheric winds derived from geostationary satellite water vapor observations. Bull. Amer. Meteor. Soc., 78, 173-195, https://doi.org/10.1175/1520-0477(1997)078<0173: UTWDFG $>2.0 . \mathrm{CO} ; 2$. 
Wang, B., and J. C. L. Chan, 2002: How strong ENSO events affect tropical storm activity over the western North Pacific. J. Climate, 15, 1643-1658, https://doi.org/10.1175/1520-0442(2002)015<1643: HSEEAT $>2.0 . \mathrm{CO} ; 2$.

_ LinHo, Y. Zhang, and M.-M. Lu, 2004: Definition of South China Sea monsoon onset and commencement of the East Asia summer monsoon. J. Climate, 17, 699-710, https:// doi.org/10.1175/2932.1.

_ , Z. W. Wu, J. P. Li, J. Liu, C. P. Chang, Y. H. Ding, and G. X. $\mathrm{Wu}, 2008$ : How to measure the strength of the East Asian summer monsoon. J. Climate, 21, 4449-4463, https://doi.org/ 10.1175/2008JCLI2183.1.

— , Y. Yang, Q.-H. Ding, H. Murakami, and F. Huang, 2010: Climate control of the global tropical storm days (1965-2008) Geophys. Res. Lett., 37, L07704, https://doi.org/10.1029/ 2010 GL042487.

Wang, Q., C. Kieu, and T.-A. Vu, 2019: Large-scale dynamics of tropical cyclone formation associated with ITCZ breakdown. Atmos. Chem. Phys., 19, 8383-8397, https://doi.org/10.5194/ acp-19-8383-2019.

Wei, N., Y. Li, D. Zhang, Z. Mai, and S. Yang, 2016: A Statistical analysis of the relationship between upper-tropospheric cold low and tropical cyclone track and intensity change over the western North Pacific. Mon. Wea. Rev., 144, 1805-1822, https://doi.org/10.1175/MWR-D-15-0370.1.

Wen, D., Y. Li, D. Zhang, L. Xue, and N. Wei, 2018: A statistical analysis of tropical upper-tropospheric trough cells over the western North Pacific during 2006-15. J. Appl. Meteor. Climatol., 57, 2469-2483, https://doi.org/10.1175/JAMC-D-180003.1

Wu, L., H. Zong, and J. Liang, 2013: Observational analysis of tropical cyclone formation associated with monsoon gyres. J. Atmos. Sci., 70, 1023-1034, https://doi.org/10.1175/JAS-D12-0117.1.

Wu, T., H. Liu, S. J. Majumdar, C. S. Velden, and J. L. Anderson, 2014: Influence of assimilating satellite-derived atmospheric motion vector observations on numerical analyses and forecasts of tropical cyclone track and intensity. Mon. Wea. Rev., 142, 49-71, https://doi.org/10.1175/MWR-D-13-00023.1.

C. S. Velden, S. J. Majumdar, H. Liu, and J. L. Anderson, 2015: Understanding the influence of assimilating subsets of enhanced atmospheric motion vectors on numerical analyses and forecasts of tropical cyclone track and intensity with an ensemble Kalman filter. Mon. Wea. Rev., 143, 2506-2531, https://doi.org/10.1175/MWR-D-14-00220.1.

Yanai, M., 1964: Formation of tropical cyclones. Rev. Geophys., 2, 367-414.

Yang, S. C., M. Corazza, A. Carrassi, E. Kalnay, and T. Miyoshi, 2009: Comparison of local ensemble transform Kalman filter, 3DVAR, and 4DVAR in a quasigeostrophic model. Mon. Wea. Rev., 137, 693-709, https://doi.org/10.1175/2008MWR2396.1.

Zhang, F., and Y. Weng, 2015: Predicting hurricane intensity and associated hazards: A five-year real-time forecast experiment with assimilation of airborne Doppler radar observations. Bull. Amer. Meteor. Soc., 96, 25-33, https://doi.org/10.1175/ BAMS-D-13-00231.1.

, J. F. Gamache, and F. D. Marks, 2011: Performance of convection-permitting hurricane initialization and prediction during 2008-2010 with ensemble data assimilation of innercore airborne Doppler radar observations. Geophys. Res. Lett., 38, L15810, https://doi.org/10.1029/2011GL048469.

Zhang, S., Z. Pu, and C. Velden, 2018: Impact of enhanced atmospheric motion vectors on HWRF hurricane analyses and forecasts with different data assimilation configurations. Mon. Wea. Rev., 146, 1549-1569, https://doi.org/10.1175/MWR-D17-0136.1.

Zhang, X., Y. Li, D. Zhang, and L. Chen, 2018: A 65-yr climatology of unusual tracks of tropical cyclones in the vicinity of China's coastal waters during 1949-2013. J. Appl. Meteor. Climatol., 57, 155-170, https://doi.org/10.1175/JAMC-D-16-0392.1.

Zhang, Z., V. Tallapragada, C. Kieu, S. Trahan, and W. Wang, 2015: HWRF based ensemble prediction system using perturbations from GEFS and stochastic convective trigger function. Trop. Cyclone Res. Rev., 3, 145-161.

Zhao, M., I. M. Held, S.-J. Lin, and G. A. Vecchi, 2009: Simulations of global hurricane climatology, interannual variability, and response to global warming using a50-km resolution GCM. J. Climate, 22, 6653-6678, https://doi.org/10.1175/2009JCLI3049.1.

Zhong, Q., J. Li, L. Zhang, R. Ding, and B. Li, 2018: Predictability of tropical cyclone intensity over the western North Pacific using the IBTrACS dataset. Mon. Wea. Rev., 146, 2741-2755, https://doi.org/10.1175/MWR-D-17-0301.1.

Zhou, L., S.-J. Lin, J.-H. Chen, L. M. Harris, X. Chen, and S. Rees, 2019: Toward convective-scale prediction within the Next Generation Global Prediction System. Bull. Amer. Meteor. Soc., 100, 1225-1243, https://doi.org/10.1175/BAMS-D-17-0246.1. 